PREPARED FOR THE U.S. DEPARTMENT OF ENERGY, UNDER CONTRACT DE-AC02-76CH03073

PPPL-3978

PPPL-3978

UC-70

Energetic Ion Transport and Concomitant Change of the Fusion Reactivity during Reconnection Events in Spherical Tori

by

Ya.I. Kolesnichenko, V.V. Lutsenko,

R.B. White, and Yu.V. Yakovenko

July 2004

NM|

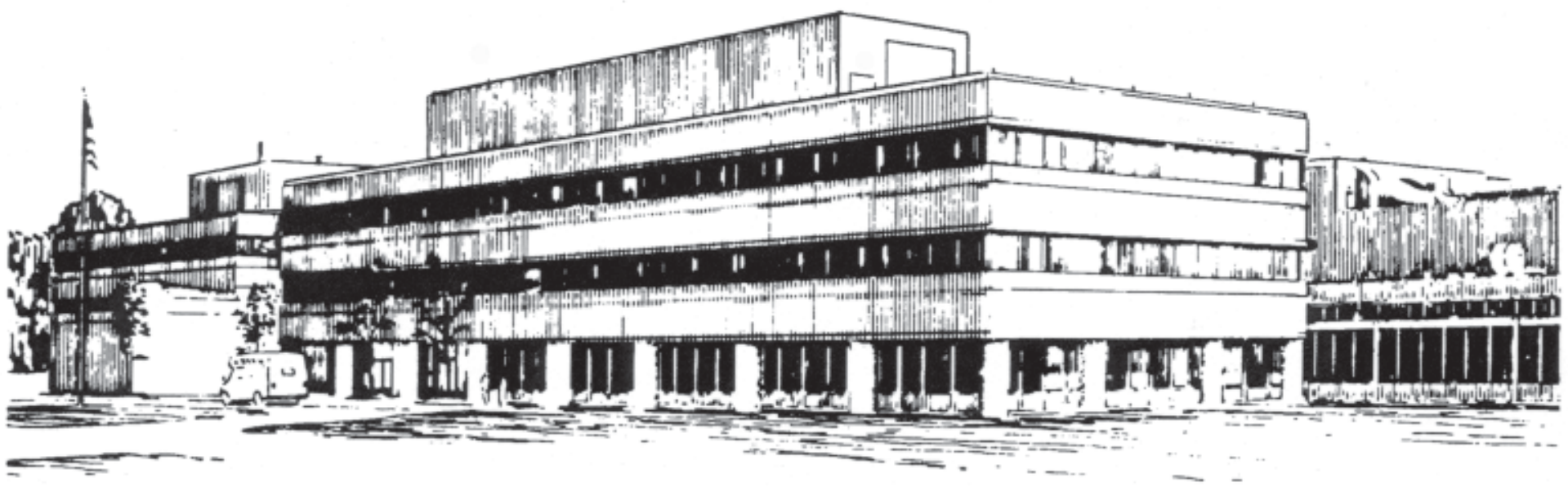

PRINCETON PLASMA PHYSICS LABORATORY PRINCETON UNIVERSITY, PRINCETON, NEW JERSEY 


\section{PPPL Reports Disclaimer}

This report was prepared as an account of work sponsored by an agency of the United States Government. Neither the United States Government nor any agency thereof, nor any of their employees, makes any warranty, express or implied, or assumes any legal liability or responsibility for the accuracy, completeness, or usefulness of any information, apparatus, product, or process disclosed, or represents that its use would not infringe privately owned rights. Reference herein to any specific commercial product, process, or service by trade name, trademark, manufacturer, or otherwise, does not necessarily constitute or imply its endorsement, recommendation, or favoring by the United States Government or any agency thereof. The views and opinions of authors expressed herein do not necessarily state or reflect those of the United States Government or any agency thereof.

\section{Availability}

This report is posted on the U.S. Department of Energy's Princeton Plasma Physics Laboratory Publications and Reports web site in Fiscal Year 2004. The home page for PPPL Reports and Publications is: http://www.pppl.gov/pub_report/

DOE and DOE Contractors can obtain copies of this report from:

U.S. Department of Energy

Office of Scientific and Technical Information

DOE Technical Information Services (DTIS)

P.O. Box 62

Oak Ridge, TN 37831

Telephone: (865) 576-8401

Fax: (865) 576-5728

Email: reports@adonis.osti.gov

This report is available to the general public from:

National Technical Information Service

U.S. Department of Commerce

5285 Port Royal Road

Springfield, VA 22161

Telephone: $1-800-553-6847$ or

(703) $605-6000$

Fax: (703) 321-8547

Internet: http://www.ntis.gov/ordering.htm 


\title{
Energetic ion transport and concomitant change of the fusion reactivity during reconnection events in spherical tori
}

\author{
Ya. I. Kolesnichenko and V. V. Lutsenko \\ Institute for Nuclear Research, Prospekt Nauky 47, Kyiv 03680, Ukraine \\ R. B. White \\ Princeton Plasma Physics Laboratory, \\ P.O. Box 451, Princeton, NJ, 08543, USA \\ Yu. V. Yakovenko \\ Institute for Nuclear Research, Prospekt Nauky 47, Kyiv 03680, Ukraine
}

(Dated: June 21, 2004)

\begin{abstract}
Effects of MHD reconnection events on the beam-plasma fusion reactivity and transport of the beam ions are studied. Based on the analysis of fusion reactivity changes induced by MHD events, the conclusion is drawn that the strong drops of the neutron yield during sawtooth crashes observed in the National Spherical Torus eXperiment (NSTX) [M. Ono et al., Nucl. Fusion 40, 557 (2000)] are associated with both a particle redistribution inside the plasma and a loss of the beam ions. Mechanisms of the energetic ion transport during sawtooth crashes are analyzed, in particular, with the use of the resonance adiabatic invariant derived in this paper. A numerical simulation of the particle motion during a sawtooth crash in NSTX is done with the code OFSEF [Ya. I. Kolesnichenko et al., Nucl. Fusion 40, 1325 (2000)] extended for a better description of the particle precession. It is shown that the motion of toroidally passing particles in NSTX can become stochastic under the influence of a crash. This stochasticity, as well as the motion along the resonance island, leads to the escape of some particles from the plasma.
\end{abstract}

PACS numbers: 52.20.Dq; 52.25.Fi; 52.35.Py; 52.55.Hc

Keywords: Spherical tori; Diamagnetism; Reconnection events; Energetic ions 


\section{INTRODUCTION}

Various forms of MHD activity affecting plasma performance were observed in Spherical Tori (ST). In particular, experiments on the National Spherical Torus eXperiment ${ }^{1}$ (NSTX) show that bursts of MHD events, such as sawtooth oscillations, Internal Reconnection Events (IRE) etc., result in strong, by a factor of two, drops of the neutron yield and signals on the Neutral Particle Analyser (NPA). ${ }^{2}$ Similar strong reduction of the neutron flux may also occur during quasi-steady-state MHD activity, but then the neutron yield decreases much slower. As it is the beam-plasma fusion reactions that mainly contribute to the neutron production, the mentioned facts indicate that the beam ions are strongly influenced by MHD activity. Therefore, a study of the energetic ions transport and concomitant change of the neutron yield in MHD-active plasmas of spherical tori is of large practical importance. In addition, the mentioned experiments have actually raised fundamental questions concerning physical mechanisms responsible for the observed phenomena. The purpose of this work is to make an attempt to elucidate the involved physics.

The structure of the work is as follows. Section II deals with the change of the beamplasma fusion reactivity during MHD events. Various patterns of the particle redistribution caused by a single crash are studied; the effect of multiple crashes is also considered. In Sec. III, mechanisms of the influence of the crash on the energetic ions are analyzed, and peculiarities of the crash-induced transport in STs are discussed. The motion of beam ions in the presence of a magnetic perturbation relevant to a nonlinear stage of the Kadomtsevtype sawtooth crash in a particular NSTX shot is studied in Sec. IV. The calculations are carried out with the code $\mathrm{OFSEF}^{3}$ (Orbit Following in the Sawtooth Electromagnetic Field) extended to model the precession of the toroidally passing particles in a non-circular plasma with finite $\beta$ ( $\beta$ is the ratio of the plasma pressure to the magnetic field pressure). The results of the paper are summarized in Sec. V. Finally, the appendices contain some material used in the analysis carried out. Appendix A contains information on the particle motion in a particular NSTX shot, the results being obtained with the code ORBIT. ${ }^{4,5}$ The derivation of a resonance adiabatic invariant of the particle motion is given in Appendix B. 


\section{MHD-EVENT-INDUCED CHANGE OF THE BEAM-PLASMA FUSION RE- ACTIVITY}

\section{A. Effect of multiple crashes on the energetic ion population}

Sawtooth oscillations and other reconnection events typically manifest themselves as relaxation oscillations of the plasma parameters. They are usually characterized by two phases, a fast phase (crash) and a slow phase (relaxation). The crash can sharply expel the energetic ions from the plasma core, leading to a change of the neutron yield of the beam-plasma reactions. When the relaxation time is about or less than the energetic ion slowing-down time, the influence of multiple crashes on the energetic ions considerably exceeds the influence of a single crash. In the contrary case, the effect is determined mainly by a single crash.

In order to see it, we note that the density of the energetic ions produced by some source switched on at the moment $t=0$ in the absence of MHD events is given by (we assume that slowing down is the only process providing the sink of the energetic ions)

$$
n_{b}(t)=n_{\infty}\left[1-\exp \left(-\frac{t}{\tau_{s}}\right)\right]+n_{0} \exp \left(-\frac{t}{\tau_{s}}\right)
$$

where $n_{0}$ and $n_{\infty}$ are the initial density and the steady-state density of the energetic ions in the absence of MHD activity, respectively, $\tau_{s}$ is the particle slowing-down time. Using Eq. (1) and assuming that all the crashes are identical, we can write the following equations for two subsequent crashes:

$$
n_{2}^{+}=n_{1}^{+}, \quad \frac{n_{2}^{+}}{n_{2}^{-}}=\delta_{s}, \quad n_{2}^{-}=n_{\infty}\left[1-\exp \left(-\frac{\Delta}{\tau_{s}}\right)\right]+n_{1}^{+} \exp \left(-\frac{\Delta}{\tau_{s}}\right),
$$

where $n_{1}^{+}$is the energetic ion density after the first crash, $n_{2}^{-}$and $n_{2}^{+}$are the energetic ion densities immediately before and after the second crash, respectively, $\Delta$ is the time between the crashes, $\delta_{s}$ is the particle density change induced by a single crash. Equations (2) yield:

$$
\frac{n_{2}^{+}}{n_{\infty}}=\delta_{s} \frac{1-\exp \left(-\Delta / \tau_{s}\right)}{1-\delta_{s} \exp \left(-\Delta / \tau_{s}\right)}
$$

We observe that $n_{2}^{+} / n_{\infty} \ll \delta_{s}$ for $\Delta \ll \tau_{s}$, and $n_{2}^{+} / n_{\infty} \approx \delta_{s}$ for $\Delta \gg \tau_{s}$.

Note that when $\Delta \ll \tau_{s}$, multiple crashes affect not only the density of the energetic ions but also the velocity distribution of these ions. As was shown, ${ }^{6}$ the distribution function, $f(v)$, becomes a saw-like function having sharp gradients (see Fig. 4 in Ref. 6). The reason 
for this is that the distribution function in the case of $\Delta \ll \tau_{s}$ retains the "memory" of multiple displacements of a given plasma volume from the plasma core to the periphery and back by repeating sawtooth events.

\section{B. Effect of a single crash: model of particles attached to flux surfaces}

In general, an MHD event affects the beam-plasma fusion reaction rate (fusion reactivity) and, thus, the neutron yield in two ways. First, it redistributes the beam and plasma particles and, second, it leads to particle loss. In conventional tokamaks, the core-localized MHD activity (such as sawtooth oscillations) typically results in a small loss of energetic ions. This may not be the case in STs. In particular, the neutron yield and NPA signals in experiments on NSTX strongly drop during IRE and sawteeth. ${ }^{2}$ Unfortunately, it is not clear whether these changes are a result of particle loss and/or their strong redistribution within the plasma (there is only one NPA line of sight, which does not allow making a tomographical reconstruction of the post-crash picture). Therefore, it is of importance to evaluate the change of the fusion reactivity in the absence of the particle loss in order to see whether the redistribution itself can explain the observed drops of the neutron yield. This will be done below.

The local beam-plasma fusion reaction rate, $I_{L}$, can be written in the following form:

$$
\begin{aligned}
I_{L} & =\int f_{b}(\mathbf{r}, \mathbf{v}) f_{i}\left(\mathbf{r}, \mathbf{v}^{\prime}\right) \sigma\left(\left|\mathbf{v}-\mathbf{v}^{\prime}\right|\right)\left|\mathbf{v}-\mathbf{v}^{\prime}\right| d^{3} \mathbf{v} d^{3} \mathbf{v}^{\prime} \approx n_{i}(\mathbf{r}) \int f_{b}(\mathbf{r}, \mathbf{v}) \sigma(v) v d^{3} \mathbf{v} \\
& \equiv n_{i}(\mathbf{r}) n_{b}(\mathbf{r})\langle\sigma v\rangle_{b i},
\end{aligned}
$$

where $f(\mathbf{r}, \mathbf{v})$ is the particle distribution function, subscripts $i$ and $b$ refer to the bulk plasma ions and the beam ions, respectively, $\sigma$ is the cross section of the fusion reaction, $n_{j}$ with $j=b, i$ is the particle density, $\langle\sigma v\rangle_{b i}=\int d^{3} \mathbf{v} f_{b} \sigma v / n_{b}$ with $n_{b}=\int d^{3} \mathbf{v} f_{b}$. Below we assume that $\langle\sigma v\rangle_{b i}$ depends only on the energy of the injected ions. This can be justified as follows. The cross-sections of both DD and DT reactions are growing functions of energy in the region of interest (below $100 \mathrm{keV}$ ). On the other hand, in STs the beam energy, $\mathcal{E}_{b}$, typically satisfies the condition $\mathcal{E}_{b} \gg \mathcal{E}_{*} \sim\left(M_{b} / M_{e}\right)^{1 / 3} T_{e}\left(T_{e}\right.$ is the electron temperature, $M$ is the particle mass), $\mathcal{E}_{*}$ in current experiments on NSTX being $20-40 \mathrm{keV}$. Therefore, Coulomb collisions between the beam ions responsible for fusion reactions and the bulk plasma ions are negligible. Thus, we can take $f_{b} \propto \mathcal{E}^{-3 / 2}$ for $\mathcal{E} \gg \mathcal{E}_{*}$ (see, e.g., Ref. 7) and neglect 
the pitch-angle dependence on $\mathbf{r}$ in $f_{b}$. Finally, neglecting the finite orbit width effects, we approximate the beam distribution function as $f_{b}=n_{b}(\mathbf{r}) F(\mathbf{v})$ and obtain that $\langle\sigma v\rangle$ does not depend on the space coordinates.

Note that the assumption that the particle orbit width is small, which we have used, is justified provided that $\Delta r_{b} \ll r_{\text {mix }}$, where $\Delta r_{b}$ is the particle orbit width, $r_{\text {mix }}$ is the mixing radius of the MHD activity. When this is the case, the particle redistribution is the strongest. In STs, $r_{\text {mix }}$ can be rather large because in many cases $r_{s} \sim a / 2\left(r_{s}\right.$ is the radius of the $q=1$ surface, $a$ is the plasma radius) and $r_{\text {mix }} \sim 1.3 r_{s}$. Therefore, in STs the mentioned condition is satisfied for the thermal ions and the beam ions with the energy $\mathcal{E} \gtrsim T_{i}$, where $T_{i}$ is the bulk ion temperature. However, it can hardly be satisfied for the most energetic injected ions $(\mathcal{E} \sim 80 \mathrm{keV})$. Nevertheless, in order to evaluate the maximum possible effect of the redistribution, we assume that the condition $\Delta r_{b} \ll r_{\text {mix }}$ is satisfied for all particles and neglect the orbital effects. Then the relative change of the fusion reactivity is completely determined by the particle density before and after the reconnection event.

In NSTX experiments the change of the global (rather than local) neutron yield is known. Therefore, below we analyze the change of the global beam-plasma fusion reactivity, $I_{G}$. Using Eq. (4), we write:

$$
\delta I_{G} \equiv \frac{I_{G}^{+}-I_{G}^{-}}{I_{G}^{-}}=\frac{\int_{0}^{x_{\text {mix }}} d x\left(n_{b}^{+} n_{i}^{+}-n_{b}^{-} n_{i}^{-}\right)}{\int_{0}^{x_{a}} d x n_{i}^{-} n_{b}^{-}},
$$

where $I_{G}=\int d^{3} \mathbf{r} I_{L}, x=r^{2} / r_{s}^{2}, x_{\text {mix }}=r_{\text {mix }}^{2} / r_{s}^{2}$, the superscripts "+" and "-" label the magnitudes before and after the MHD event, respectively. In order to know the post-crash radial profiles, we have to specify the character of the MHD activity and its effect on the particle transport.

We consider an event accompanied by the reconnection of magnetic field lines. In this case, the process can be described by the Kadomtsev model of the sawtooth crash. $^{8}$ Admittedly, the mentioned model predicts that the post-crash central safety factor, $q_{0}^{+}$, equals to unity, which often contradicts to experimental data. There exists also another model based on the same idea of complete reconnection during the crash, which is consistent with experiments with $q^{+}(0)<1 .{ }^{9}$ However, the model ${ }^{9}$ leads to a smaller change of the neutron yield (although it provides stronger redistribution of the particles). ${ }^{10}$ Therefore, to evaluate the maximum possible change of the neutron yield, we will use the Kadomtsev model.

In this subsection, we assume that both beam and plasma ions are redistributed by the 
crash because they are attached to the evolving flux surfaces. Then we can express $\delta I_{G}$ in terms of the radial profiles of $n_{i}$ and $n_{b}$ inside the $q=1$ radius before the crash as follows: ${ }^{10}$

$$
\delta I_{G}=-\frac{\int_{0}^{1} d x_{1}^{-}\left(n_{b 1}^{-}-n_{b 2}^{-}\right)\left(n_{i 1}^{-}-n_{i 2}^{-}\right) V_{2} /\left(V_{1}+V_{2}\right)}{\int_{0}^{x_{a}} d x^{-} n_{b}^{-} n_{i}^{-}}
$$

where the subscripts "1" and "2" are relevant to the regions $r<r_{s}^{-}$and $r>r_{s}^{-}$, respectively, $V_{1,2}=\left|\iota\left(x_{1,2}\right)-1\right|^{-1}$ are the effective volumes of the layers inside and outside $r_{s}^{-}$involved to the reconnection process, $x_{a}=a^{2} / r_{s}^{2}, n_{1}^{-} \equiv n\left(x_{1}^{-}\right), n_{2}^{-} \equiv n\left(x_{2}^{-}\right), x_{2}^{-}=x_{2}^{-}\left(x_{1}^{-}\right)$, the function $x_{2}^{-}\left(x_{1}^{-}\right)$is determined from the condition that the magnetic reconnection mixes the particles located at the radii with the same magnitude of the helical magnetic flux $\left(\psi_{h}\right)$, i.e., $\psi_{h}\left(x_{2}^{-}\right)=\psi_{h}\left(x_{1}^{-}\right)$with $\psi_{h}(r)=R_{0} \int_{0}^{r} B_{p}(1-q) d r$ ( $B_{p}$ is the poloidal magnetic flux, $R_{0}$ is the large radius of the torus) in the axisymmetric states before and after the crash.

To find the dependence of $x_{2}^{-}$on $x_{1}^{-}$, we take the safety factor (or the rotational transform, $\iota=q^{-1}$ ) before an MHD event in the form:

$$
\iota\left(x_{1,2}^{-}\right) \equiv \frac{1}{q\left(x_{1,2}^{-}\right)}=1+\Upsilon_{1,2}\left(1-x_{1,2}^{-}\right),
$$

where

$$
\Upsilon_{1}=\iota_{0}^{-}-1, \quad \Upsilon_{2}=\frac{1-\iota_{a}}{x_{a}-1}
$$

with $\iota_{a} \equiv \iota(a)$. When $q(r)$ is given by Eq. (7), we have:

$$
x_{2}^{-}=1+\left(x_{\text {mix }}-1\right)\left(1-x_{1}^{-}\right), \quad V_{2}=\left(x_{\text {mix }}-1\right) V_{1}, \quad x_{\text {mix }}=1+\sqrt{\frac{\Upsilon_{1}}{\Upsilon_{2}}} .
$$

Equation (7) can provide a reasonable approximation for the safety factor profile in NSTX shots. In particular, when $\Upsilon_{1}=0.11, \Upsilon_{2}=0.39$, and $r_{s}^{2} / a^{2}=0.3$, it yields $q_{0}=0.9$, $q_{a}=10$, and the $q(r)$ profile close to that in the NSTX shot \#104505 (where sawtooth crashes strongly affected the beam ions). Note that in the considered case the sawtooth mixing radius determined by Eq. (9) is $x_{\text {mix }}=1.53$.

Using Eq. (9) and taking

$$
n_{b, i}=\left(1-\frac{x}{x_{a}}\right)^{\nu_{b, i}}
$$

we find:

$$
\delta I_{G} \approx-\nu_{b} \nu_{i}\left(\nu_{b}+\nu_{i}+1\right) \frac{x_{\mathrm{mix}}\left(x_{\mathrm{mix}}-1\right)}{3 x_{a}^{3}} .
$$

This expression was obtained in the assumption that $n_{b, p}(x)$ can be approximated as $n_{b, p}(x)=n_{b, p}(0)+n_{b, p}^{\prime} x$ with $n^{\prime}=d n /\left.d x\right|_{x=0}$. We observe that, as expected, $\delta I$ grows 
with $r_{\text {mix }}$. A simple analysis shows that it grows also with $r_{s}$ in all cases of interest: the function $y\left(r_{s}\right) \equiv x_{\text {mix }}\left(x_{\text {mix }}-1\right) / x_{a}^{3}$ has a maximum in a point very close to $a$ (for instance, the maximum is located at $r_{s} \approx 0.9 a$ for $\Upsilon_{1}=0.11$ and $\left.\Upsilon_{2}=0.39\right)$. However, these results are valid only for relatively flat profiles, $\left|n_{j}^{\prime} x_{\text {mix }} / n_{j}(0)\right|<1$, which in the particular case of the safety factor given by Eq. (7) with the parameters mentioned above leads to $\nu_{j} \lesssim 2$. Taking $\nu_{b}=2$ and $\nu_{i}=0.5-1$, we obtain from Eq. (11) that $\delta I_{G}=2.5-5.8 \%$. However, the beam profile is often more peaked $\left(\nu_{b}>2\right)$. Therefore, we consider the contrary limit case, taking $n_{b} \propto \delta(x)$. Then one can easily obtain from Eqs. (6) and (10) that (cf. Ref. 10)

$$
\delta I_{G}=-\frac{x_{\mathrm{mix}}-1}{x_{\mathrm{mix}}}\left[1-\left(1-\frac{x_{\mathrm{mix}}}{x_{a}}\right)^{\nu_{i}}\right] .
$$

Equation (12) leads to $\left|\delta I_{G}\right|=9 \%$ for $\nu_{i}=1$, and $\left|\delta I_{G}\right|=16 \%$ for $\nu_{i}=0.5$, which is still much less than the drops of the neutron yield observed experimentally on NSTX.

C. Effect of a single crash: model with the energetic ions not sensitive to the crash

It is known that most trapped particles with the energy exceeding a certain critical magnitude, $\mathcal{E}_{\text {crit }}^{\text {trapped }}$, are not sensitive to the reconnection events unless $\beta$ is very large (the marginally trapped particles are an exception). ${ }^{11}$ The critical energy is given by ${ }^{3,12}$

$$
\mathcal{E}_{\text {crit }}^{\text {trapped }}=2 \pi M k_{s} r_{s} R_{0} \omega_{B} / \tau_{\text {crash }}
$$

where $\tau_{\text {crash }}$ is the crash duration, $k$ is the elongation of the plasma cross-section, the subscript $s$ labels the values at the $q=1$ surface, $\omega_{B}$ is the particle gyrofrequency. One can see from Eq. (13) that the magnitude of the critical energy in STs is rather low. Therefore, the population of well-trapped energetic ions is not sensitive to the reconnection events in the discharges with moderate $\beta\left(\beta(0)<r_{s} / R_{0}\right)$ relevant to current experiments on NSTX. Thus, it may be of interest to calculate the change of the neutron yield in the assumption that the bulk plasma ions are redistributed according to the Kadomtsev model of the sawtooth crash, whereas the energetic ion population is not redistributed.

One can show that the pre-crash coordinates can be expressed through the post-crash coordinates in the Kadomtsev model as follows:

$$
x_{1}^{-}=1-\frac{x^{+}}{x_{m}}, \quad x_{2}^{-}=1+x^{+}\left(1-\frac{1}{x_{\text {mix }}}\right) .
$$


Using these equations, we obtain the following post-crash profile of the bulk ions having the pre-crash profile given by Eq. (10):

$$
n^{+}=\frac{n_{0}}{x_{\operatorname{mix}}}\left(\left[1-\frac{1}{x_{a}}\left(1-\frac{x^{+}}{x_{\operatorname{mix}}}\right)\right]^{\nu_{i}}+\left(x_{\operatorname{mix}}-1\right)\left\{1-\frac{1}{x_{a}}\left[1+x^{+}\left(1-\frac{1}{x_{\operatorname{mix}}}\right)\right]\right\}^{\nu_{i}}\right) .
$$

Below we take $\nu=1$, in which case

$$
n^{+}=n_{0}\left[1-\frac{1}{x_{a}}+\left(\frac{2}{x_{\operatorname{mix}}}-1\right) \frac{x^{+}}{x_{a}}\right] .
$$

Now we can calculate the change of the neutron yield:

$$
\delta I_{G}=\frac{\nu_{b}+2}{\nu_{b}+1}\left(\frac{2}{x_{\operatorname{mix}}}-\frac{1}{x_{a}}\right)\left(1-x_{a m}^{\nu_{b}+1}\right)-\frac{2}{x_{\operatorname{mix}}}\left(1-x_{a m}^{\nu_{b}+2}\right)
$$

where $x_{a m} \equiv 1-x_{\text {mix }} / x_{a}$. It follows from Eq. (17) that the drop of the neutron flux grows with $\nu$ (vanishing for $\nu_{b}=0$ ). When $\nu_{b} \rightarrow \infty$

$$
\delta I_{G} \rightarrow-\frac{1}{x_{a}}
$$

which for the safety factor used above yields $\delta I_{G}=-30 \%$. However, the change of the neutron flux for realistic beam distributions is considerably less. For instance, taking $\nu_{b}=6$, we obtain $\delta I_{G}=17 \%$.

\section{Effect of a single crash: model with flat post-MHD profiles}

The analysis above was made in the assumption that a sawtooth crash of the Kadomtsev type occurs and either only the plasma or both the beam and the plasma are attached to the flux surfaces. Below we make an estimate which does not depend on the character of the MHD event. The main assumption we need is that the MHD event strongly mixes, at least, one component of the beam-plasma system, so that $n_{b}^{+}(r)=$ const or $n_{i}^{+}(r)=$ const or the particle densities of both the beam and plasma are flat immediately after the MHD event. This may be the case, e.g., when the energetic ions are redistributed by the MHD event inside the mixing radius but reach the plasma edge due to their finite orbit width. Furthermore, the assumption that $n_{j}(r)=$ const is justified even when the MHD event results in a flat radial profile of the plasma/beam particles only at $r<r_{\text {mix }}$, provided that $r_{\text {mix }}$ is so large that the neutron production in the periphery region, $r>r_{\text {mix }}$, is negligible. 
Let us introduce the particle number $N_{j}=\int_{0}^{a} d x n_{j}(x)$ and assume that $n_{b}^{+}(r)=$ const. Then, using Eq. (4), we obtain:

$$
\frac{I_{G}^{+}}{I_{G}^{-}}=\frac{N_{b}^{+} N_{i}^{+}}{x_{a} \int_{0}^{x_{a}} d x^{-} n_{b}^{-} n_{i}^{-}} .
$$

Note that this expression is symmetric with respect to the subscripts $b$ and $i$; therefore, it is valid not only for $n_{b}^{+}(r)=$ const but also for $n_{i}^{+}(r)=$ const. If the number of the particles is conserved, Eq. (19) determines the drop of the neutron yield, provided that the pre-crash profiles are known.

Let us assume that $N_{i}^{+}=N_{i}^{-}$(an MHD event perturbs only the plasma core, thus producing no loss of the thermal ions), but we will not specify $N_{b}^{+}$. Then, taking the radial profiles in the form given by Eq. (10), we obtain:

$$
\begin{gathered}
N_{i}^{+}=n_{i}^{-}(0) x_{a} /\left(\nu_{i}+1\right), \\
\int_{0}^{x_{a}} d x n_{b}^{-} n_{i}^{-}=N_{b}^{-} n_{i}(0) \frac{\nu_{b}+1}{\nu_{b}+\nu_{i}+1} .
\end{gathered}
$$

Using these equations, we find that

$$
\frac{I_{G}^{+}}{I_{G}^{-}}=\frac{N_{b}^{+}}{N_{b}^{-}} \frac{\left(\nu_{b}+\nu_{i}+1\right)}{\left(\nu_{b}+1\right)\left(\nu_{i}+1\right)} .
$$

Equation Eq. (22) shows that, as in the cases described in the previous sections, the largest drop of the neutron yield occurs when the beam ions have a very peaked pre-crash profile. For $\nu_{b} \rightarrow \infty$ we have:

$$
\frac{I_{G}^{+}}{I_{G}^{-}}=\frac{N_{b}^{+}}{N_{b}^{-}} \frac{1}{\left(\nu_{i}+1\right)},
$$

It follows from Eq. (23) that $I_{G}^{+} / I_{G}^{-}=0.5$ for $N_{b}^{+}=N_{b}^{-}$and $\nu_{i}=1$. This corresponds to the change of the neutron yield observed in NSTX. However, this estimate was obtained for unrealistic assumptions on the particle profiles. For any realistic profiles we have to use Eq. (22). Taking again $I_{G}^{+} / I_{G}^{-}=0.5$ and assuming $\nu_{b}=6, \nu_{i}=0.5$, we obtain from Eq. (22) that $N_{b}^{+} / N_{b}^{-}=0.7$, which implies that $30 \%$ of the beam ions are lost. 


\section{MECHANISMS OF THE PARTICLE REDISTRIBUTION DURING MHD EVENTS}

\section{A. Qualitative analysis}

The sawtooth effect on fast particles in conventional tokamaks has been extensively studied both experimentally and theoretically (see, e.g., the review in Ref. 3 and references therein). The results of theoretical works ${ }^{3,12-15}$ can be briefly summarized as follows. The sawtooth-induced particle redistribution is a result of competition of several processes. First, the electric field associated with the plasma flow tends to move any particle together with the flow. The characteristic time of this motion is $\tau_{\text {crash }}$, the sawtooth crash duration. Second, the toroidal precession tends to move a particle so that its radial coordinate, $r$, is constant and, hence, to prevent the particle redistribution. Third, the longitudinal motion tends to move particles along the displaced flux surfaces. The characteristic time of this motion is the period of the particle trip around a flux surface,

$$
\tau_{L}=\frac{2 \pi R_{0}}{\left\langle v_{\|}\right\rangle\left|q_{0}^{-1}-1\right|},
$$

where $v_{\|}$is the particle velocity along the magnetic field, and $q_{0}$ the safety factor at the axis. (Here we restrict ourselves to considering "conventional" sawteeth caused by $m=$ $n=1$ perturbations, where $m$ and $n$ are the poloidal and toroidal mode numbers, although sawteeth arising due to perturbation with different values of $m$ and $n$ can be considered in the same approach.) The resulting particle motion depends on the relative characteristic times of the three processes, which, in turn, depend on the particle energy and pitch angle. The passing particles with the orbit widths less than $r_{\text {mix }}$ are typically characterized by fast longitudinal motion and relatively slow precession; therefore, they are strongly redistributed by a crash. The longitudinal motion of the trapped particles is negligible. Therefore, the intensity of their redistribution depends on their energy: When the energy exceeds the critical value determined by the condition $\tau_{\text {crash }}=\tau_{\text {pr }}$, where $\tau_{\text {pr }}$ is the precession period, [which leads to Eq. (13)] the precession is fast enough to prevent the redistribution. ${ }^{12,13}$ Finally, the precession and bounce periods of the particles with large orbit widths $\left(\sim r_{\text {mix }}\right)$ are typically of the same order of magnitude. Therefore, the precession is able to prevent the redistribution, and such particles can be redistributed only due to resonances between their precession and bounce motion. ${ }^{14,15}$ 
Low aspect ratio and strong plasma diamagnetism in spherical tori modify this pattern. First, in high- $\beta$ plasmas of modern spherical tori, the particle precession has a strong diamagnetic component. When the magnetic configuration is perturbed by a crash, this component tends to move particles along the surfaces of constant pressure, which are the displaced flux surfaces; therefore, it promotes the particle redistribution instead of preventing it. ${ }^{11}$ Second, the relative magnitudes of the characteristic times of the mentioned processes are quite different. Let us consider $80-\mathrm{keV}$ passing injected deuterons in NSTX. Assuming that $\left|q^{-1}-1\right| \sim 0.1, v_{\|} / v=0.8, R_{0} \sim 100 \mathrm{~cm}$, we find: $\tau_{L} \sim 3 \times 10^{-5} \mathrm{~s}$. For a parabolic pressure profile the diamagnetic precession period can be estimated as [see Eq. (31)] $\tau_{\text {dia }} \sim 2 \pi a^{2} /\left(v \rho_{\perp} \beta_{0}\right) \sim 10^{-4} \mathrm{~s}$, where $\rho_{\perp}=v_{\perp} / \omega_{B}, \omega_{B}$ is the deuteron cyclotron frequency, $a=68 \mathrm{~cm}, B_{0}=0.3 \mathrm{~T}$, and $\beta_{0}$ is $\beta$ at the magnetic axis, which is taken to be $11 \%$ for this estimate. The period of the "conventional" precession caused by toroidicity can be estimated as $^{16}$ [see Eq. (A1)]

$$
\tau_{\mathrm{pr}}=\frac{2 \pi R_{0}^{2}}{\xi v \rho} \sim 10^{-5} \mathrm{~s}
$$

where $\rho=v / \omega_{B}$, and $\xi$ is a certain dimensionless parameter determined by the pitch angle and properties of the magnetic configuration, which was assumed to be $\sim 1$ for this estimate. As $\tau_{\text {crash }} \sim 10^{-4} \mathrm{~s}$ in most tokamaks (unfortunately, we have no such data concerning spherical tori yet), we observe that the precession, which prevents the particle redistribution, is faster than the rest of factors. Hence, in contrast to conventional tokamaks, the passing fast deuterons in NSTX are not expected to follow the evolving flux surfaces, and one needs to involve some resonance phenomena to explain their redistribution or loss. However, this conclusion may be wrong for particles with lower energy. In general, one can conclude that $\tau_{\text {pr }}, \tau_{L} \ll \tau_{\text {dia }}, \tau_{\text {crash }}$.

Therefore, the general pattern of the motion of passing particles is determined mainly by the competition of longitudinal motion and precession. As the energy dependence of the characteristic times of these processes is different, $\tau_{L} \propto \mathcal{E}^{-1 / 2}$ and $\tau_{\mathrm{pr}} \propto \mathcal{E}^{-1}$, we recover the conclusion ${ }^{12}$ that there exists a critical energy for the redistribution of passing particles, $\mathcal{E}_{\text {crit }}^{\text {passing }}$, determined by the relationship $\tau_{L}=A \tau_{p r}$, where $A$ is a dimensionless constant determined by the process geometry. Our calculations presented below (Subsec. III B) show that $A \approx 1 / 8$. The particles with the energy below the critical one are strongly redistributed during the crash, whereas for higher energies the crash affects mainly the particles within 
the resonance island. From Eqs. (24) and (25) we obtain:

$$
\mathcal{E}_{\text {crit }}^{\text {passing }}=\frac{M}{2}\left(\frac{R_{0} \omega_{B 0}}{8 \xi}\langle\chi\rangle\left|q_{0}^{-1}-1\right|\right)^{2},
$$

where $\chi=v_{\|} / v,\langle\ldots\rangle$ denotes bounce averaging. In particular, $\mathcal{E}_{\text {crit }}^{\text {passing }}=\left(\langle\chi\rangle\left|q_{0}^{-1}-1\right| / \xi\right)^{2}$. $50 \mathrm{keV}$ in NSTX. Thus, the energy of the ions responsible for the neutron yield considerably exceeds both $\mathcal{E}_{\text {crit }}^{\text {trapped }}$ and $\mathcal{E}_{\text {crit }}^{\text {passing }}$ when $|\xi| \gtrsim 0.2$. This means that these particles can be redistributed only due to resonance effects.

One can assume a priopi that the motion of passing particles during a sawtooth event is determined to a large extent by the resonance $\omega_{\phi} / \omega_{\theta}=1 / 1$, where $\omega_{\phi}$ and $\omega_{\theta}$ are the frequencies of the poloidal and toroidal motion, respectively: $\omega_{\phi} \approx \omega_{\theta}$ for most circulating particles in the region of sawteeth, and the $m=n=1$ harmonic dominates in the sawtooth instability. This resonance is likely to form resonance islands near the resonant drift surface, which will cause redistribution of particles or even loss if the island reaches elements of the device hardware. Even some particles outside the island can be lost because of the distortion of their drift surfaces by the perturbation.

However, as we will see in Sec. IV, the particle motion is stochastic in many cases. The main difficulty with understanding the origin of such stochasticity is that $\omega_{\phi} / \omega_{\theta}=s / n$ is typically slightly below unity in the central part of the plasma (see Fig. 6). Therefore, to explain the appearance (and subsequent overlap) of resonance islands, one has to involve resonances $\omega_{\phi} / \omega_{\theta}=s / n$ with $n \gg 1$, which, in turn, require perturbation harmonics with the toroidal number $n \gg 1$ to be present. One possible mechanism of the realization of such resonances is the appearance of higher harmonics of the perturbation on the nonlinear stage (in particular, sideband resonances $\omega_{\phi} / \omega_{\theta}=s / n$ with the harmonics $m=n \neq 1$ are possible $\left.{ }^{14}\right)$. Another possible mechanism is associated with the fact that particles with small poloidal action undergo relatively large radial displacements under an $m=n=1$ perturbation. As a result, additional Fourier harmonics appear in the Fourier spectrum of the perturbation in the canonical coordinates associated with the displaced drift surfaces. This can cause global stochasticity when the perturbation exceeds a certain critical level. ${ }^{15}$

One can expect that relatively high diamagnetism in spherical tori will provide an additional mechanism of the particle motion stochasticity. The matter is that a plasma redistribution in this case is accompanied by a noticeable variation of the magnetic field strength (i.e., the longitudinal component of the magnetic perturbation is not small). The magnetic 
reconnection, in particular, will create large pressure gradients on the boundaries of the reconnected and unreconnected plasma. The effect of such a perturbation on the particles that cross these boundaries owing to their orbital motion and/or precession will consist in transversal "kicks" of the $\nabla B$ drift. As the Fourier spectrum of such perturbations of the particle motion is very rich, one can expect the appearance of numerous islands of resonances $\omega_{\phi} / \omega_{\theta}=m / n$ with large numbers $m$ and $n$. The numerical calculations taking account of the effect of the large pressure gradients arising during a magnetic reconnection indeed show that finite $\beta$ increases the region of stochasticity. ${ }^{17}$

\section{B. The $1 / 1$ resonance}

We begin with studying the bounce-averaged resonant motion, using the adiabatic invariant of the resonant motion. The drawbacks of the bounce-averaged description are that it enables one to study only a chosen single resonance and that stochastic motion can be detected only indirectly, from the overlap of different resonances. Nevertheless, it gives a possibility to evaluate the resonant response of particles much easier. The invariant that we will use is derived in Appendix B. It is a generalization of a previous result ${ }^{18}$ to the case of perturbations affecting the magnetic field strength, $B$ (see also Ref. 19).

We will assume that the crash duration is sufficiently large and the perturbation frequency is sufficiently small to consider the perturbation as static when studying the particle motion (our estimates in Subsec. III A partly justify this assumption). Then, as shown in Appendix B, the guiding-center motion near the 1/1 resonance possesses the following adiabatic invariant:

$$
\begin{aligned}
I\left(v, \mu, J_{\phi}, \phi\right) & =J_{\theta}+J_{\phi} \\
& +\frac{1}{2 \pi} \oint_{L\left(v, \mu, J_{\phi}, \phi\right)}\left[d \mathbf{x} \cdot\left(\frac{e}{c} \tilde{\mathbf{A}}+M \frac{v_{\|}}{B} \tilde{\mathbf{B}}-M \frac{v_{\|}}{B^{2}} \mathbf{B} \tilde{B}\right)-d t(\mu \tilde{B}+e \Phi)\right],
\end{aligned}
$$

where $\mathbf{A}$ and $\Phi$ are the vector and scalar potential of the electromagnetic field; tildes refer to the perturbation; $\mathbf{x}$ is the location of the guiding center; $J_{\phi}$ is the canonical angular momentum; the poloidal action $J_{\theta}$ is defined by

$$
J_{\theta}\left(J_{\phi}, v, \mu\right)=\frac{1}{2 \pi} \oint d \mathbf{x} \cdot\left[\frac{e}{c} \mathbf{A}(\mathbf{x})+\frac{M v_{\|}}{B} \mathbf{B}(\mathbf{x})\right],
$$

the integration in Eq. (28) being performed along the poloidal contour $J_{\phi}=$ const, $v=$ const, $\mu=$ const. The contour $L\left(v, \mu, J_{\phi}, \phi\right)$ in Eq. (27), along which the perturbation is integrated, 
is the unperturbed trajectory of a particle with the given magnitudes of $v, \mu$, and $J_{\phi}$ with the toroidal frequency "corrected" to satisfy the resonance exactly [to make $L\left(v, \mu, J_{\phi}, \phi\right)$ a closed loop], i.e.,

$$
\left.\mathbf{v}\right|_{L}=\left.\mathbf{v}\right|_{0}+\left(\omega_{\theta}-\omega_{\phi}\right) \nabla \phi
$$

where the subscripts " $L$ " and " 0 " refer to the loop $L$ and the actual unperturbed trajectory, respectively. The integral in Eq. (27) depends on the toroidal position of the loop L. For definiteness, we assume that $L\left(v, \mu, J_{\phi}, \phi\right)$ starts from that point in the equatorial plane of the torus $(\theta=0$ or $\pi)$ with the toroidal angle $\phi$ which is at the maximum distance from the axis of symmetry $(R)$.

When the perturbation is steady-state or varies sufficiently slowly, the bounce-averaged trajectories of particles coincide with the level contours of the invariant given by Eq. (27). As discussed below, the crash duration in NSTX well exceeds the characteristic times of the motion of the passing particles. Thus, we can use the invariant to study the effect of the $1 / 1$ resonance on the motion of such particles.

Assuming that the crash is caused mainly by an $m=n=1$ electromagnetic perturbation, we write the vector potential of the perturbation as follows:

$$
\tilde{\mathbf{A}}=-\Psi_{h}(r, \alpha) \nabla \phi-F(r, \alpha) \nabla \alpha
$$

where $\alpha=\theta-\phi, \Psi_{h}$ is the helical magnetic flux responsible for the transversal part of the perturbation (the flux surfaces under the perturbation are the level surfaces of $\Psi_{h}$ ), $F$ describes the diamagnetic perturbation of $B$ and is determined by $\Psi_{h}$ and the pre-crash distribution of $p$. To model $\Psi_{h}$, we use the analytical expressions suggested in Ref. 12, which describe the configuration evolving in agreement with the Kadomtsev model ${ }^{8}$ of the sawtooth crash. Concerning the diamagnetic perturbation, we assume that it is "attached" to $\Psi_{h}$ (see Ref. 11 for details).

We assume that the projection of the integration loop given by Eq. (29) onto the plane $(r \cos \alpha, r \sin \alpha)$ can be approximated by a circle with the radius $\Delta r$, where $\Delta r=v_{D} /\left(k \omega_{\theta}\right)$ is the radial excursion of a particle orbit. One can show that the projections of the orbits of well circulating particles indeed have this shape in the central region of the plasma. At the periphery the orbit shape is more complicated, but one can expect that this simplifying assumption does not affect our results strongly. Thus, the problem is reduced to averaging the perturbation over circles in the plane $(r \cos \alpha, r \sin \alpha)$ and drawing the level contours 
of the resulting function. This is still too cumbersome to be done analytically for the perturbation model we use, but the numerical realization of this scheme is fast and simple.

Using this method, we have studied the dependence of the particle motion on parameters. The general pattern of the motion turns out to depend mainly on the relative magnitudes of the precession and longitudinal terms in Eq. (27). Figure 1 shows the drift surfaces during a Kadomtsev reconnection for two different magnitudes of $\xi$. For $\xi=-0.01$ [Fig. 1 (a)], which corresponds to $A \equiv \tau_{L} / \tau_{\mathrm{pr}}=0.02$, the perturbed drift surfaces almost coincide with the flux surfaces. Just as the latter, they are reconnected during the crash, which results in a redistribution of the particles inside the sawtooth mixing radius, but the particles orbits at the periphery are almost not affected. The $1 / 1$ resonance island almost coincides with the magnetic island and covers the plasma center. The opposite limit case of strong precession is shown in Fig. 1 (b) $(\xi=-0.2, A=0.4)$. Here the deviations of the particle orbits from $r=$ const are much less, but the $1 / 1$ resonance island has shifted outwards. For $A \approx 1 / 8$ the $1 / 1$ resonance island marginally reaches the plasma center. It is reasonable to consider that this corresponds to the critical energy of passing particles, as was assumed in Subsec. III A.

To study how the particle redistribution is affected by diamagnetism, we have made similar calculations with $\chi$ varying between 0.6 and 1 and $\beta_{0}$, between 0 and 0.3 . We have found that the dependence of the island width on these parameters is negligible, although the displacement of the central drift surfaces is sensitive to $\beta_{0}$.

The dependence of the bounce-averaged particle motion on the orbit width also turned out to be weak as long as the orbit width is less than the mixing radius.

A general conclusion from this analysis is that regular motion due to the $1 / 1$ resonance flattens the radial profile of beam ions. It can lead to significant losses of beam ions during sawteeth when the island width and the mixing radius are sufficiently large.

\section{NUMERICAL STUDY OF THE SAWTOOTH-CRASH-INDUCED MOTION OF THE BEAM IONS IN NSTX}

In this section we study the influence of sawtooth crashes on the beam ions in a particular NSTX shot. We have selected the shot \#104505, where several MHD events were observed, some of which were accompanied by strong drops of the NPA signals (the measured particle energies were $70 \mathrm{keV}, 53 \mathrm{keV}$, and $45 \mathrm{keV}$ ) and the neutron flux. ${ }^{2}$ As was shown above, such 
strong drops (50\% and more) are evidences of the strong influence of the MHD events on beam ions. The earliest two events (at $t_{1} \approx 0.16 \mathrm{~s}$ and $t_{2} \approx 0.19 \mathrm{~s}$ ), most probably, can be identified as sawtooth crashes rather than IRE, which is confirmed by the absence of noticeable current spikes and the availability of $n=1$ signals in Mirnov spectrograms. The crashes occurred during steady-state Neutral Beam Injection (NBI) of 80-keV deuterons when $q_{0}$ was slowly decreasing. The $q(r)$ profiles calculated by the code TRANSP $^{20}$ for instants preceding the two crashes $(t=0.155 \mathrm{~s}$ and $t=0.185 \mathrm{~s})$ are given in Fig. $2 .{ }^{21}$ We observe that $q_{0}$ is above unity $\left(q_{0}=1.2\right)$ before the first crash and slightly below unity $\left(q_{0}=0.99\right)$ before the second one, which seems to contradict the identification of the two events as sawteeth. However, the uncertainty in the reconstruction of $q_{0}$ by TRANSP is rather high. Therefore, we suppose that the two events are sawteeth and consider the precrash value of $q_{0}$ as a parameter in the range of $0.8 \leq q_{0} \leq 1$. Other relevant plasma parameters were $B_{0}=0.36 \mathrm{~T}, \beta_{0}=11 \%$.

TRANSP calculations ${ }^{21}$ show that the population of beam ions in the shot \#104505 consists mainly of particles with large longitudinal velocity $\chi>0.5$. Calculations with the code ORBIT show that such particles are toroidally passing (i.e., $v_{\|}$does not change the sign during their orbital motion, although turning points $d \theta / d t=0$ can exist, i.e., the particles can be "semi-trapped"). Taking this into account, below we concentrate on particles with relatively small $\lambda$.

We assume that the crash duration considerably exceeds the particle transit period (the latter is about $3 \times 10^{6} \mathrm{~s}$ ). Then the electromagnetic perturbation relevant to the crash is approximately constant for many transit periods. This justifies the study of the particle motion in a steady-state magnetic configuration corresponding to a certain stage of the crash, which will be done below.

In order to study the crash-induced particle transport, we use the code OFSEF. ${ }^{3}$ This code calculates the particle orbits in a magnetic configuration modeling the Kadomtsev type of the sawtooth crash. It supposes that the plasma equilibrium before and after the crash is rather simple (elliptic flux surfaces, no Shafranov shift, no paramagnetic and diamagnetic effects on the magnetic field strength). Because of this, the code underestimates the precession rate. Therefore, we have modified this code by adding terms modeling the precession. First of all, we included effects of the plasma pressure on the particle drift motion (the "diamagnetic precession"): the finite pressure terms may play an important role even for moderate $\beta$ 
because large local pressure gradients appear during the reconnection. This was done by introducing the additional diamagnetic drift given by (cf. Ref. 11)

$$
\mathbf{v}_{\text {dia }}=-\frac{4 \pi \mu}{M B^{2} \omega_{B}} \mathbf{B} \times \nabla p
$$

to the particle motion, where $\mu$ is the particle magnetic moment. In addition to this drift, the particles undergo the "conventional precession", i.e., the precession not associated with pressure gradients. One can expect that such precession is weakly affected by magnetic perturbations (even the poloidal magnetic field weakly changes because of a typical sawtooth crash) and tends to move the particles across the perturbed flux surfaces. ${ }^{11,12}$ To take account of such precession in OFSEF, we calculated the precession of various $80-\mathrm{keV}$ deuterons with the Hamiltonian guiding-center code ORBIT, ${ }^{4,5}$ using realistic equilibria of the shot \#104505 (see Appendix A). The results of these calculations were considered as the conventional precession and incorporated into the code OFSEF. However, we should note that the study of the precession in the unperturbed state is not sufficient for the real description of the precession during the crash. The matter is that the magnetic configuration is strongly non-axisymmetric during the crash. In addition, as follows from an analytical formula for the precession frequency [Eq. (A2)], the role of the terms that depend on the plasma pressure in the mentioned formula is quite considerable and can even be dominant in the shot \#104505. This implies that the ORBIT calculations overestimate the role of the conventional precession (it is not possible to subtract the precession caused by the pressure) and does not take into account the peculiarities associated with the absence of the axial symmetry during the crash. For this reason, we investigated the particle transport with OFSEF modified as described above for two cases: with the ORBIT precession term (which overestimates the conventional precession) and without it (which underestimates the conventional precession).

We studied the particle motion by calculating Poincaré maps at a certain (non-linear) phase of the crash for various $q_{0}$. Because the perturbation amplitude depends on $q_{0}$ (the transversal component of the magnetic field perturbation is proportional to $|1-q|$ inside the $q=1$ radius for small $\beta$ ), the cases with $q_{0}$ approaching unity correspond to weaker perturbations. Therefore, as expected, we found that the particle motion is mainly regular when $q_{0}$ is sufficiently close to unity (see Fig. 3, where the results of calculations for $q_{0}=$ 0.95 are presented). Being mainly regular, the pictures in Fig. 3 (a) and Fig. 3 (b) are, nevertheless, different. There is a wide resonance island in the absence of the conventional 
precession term, and the particle motion is strongly perturbed. In contrast to this, the presence of the conventional precession term leads to motion with the radial coordinate being almost constant, and the resonance island is not noticeable.

A decrease of $q_{0}$ increases the island width and the stochasticity domain. In particular, the stochastic region dominates for $q_{0}=0.8$ when the precession is negligible [Fig. 4 (b)]. When the conventional precession is on [Fig. 4 (a)], the island width is smaller, but the island is shifted outwards, which agrees with the qualitative consideration in Subsec. III B. The shown results are relevant to particles with $\lambda=0.58$. The results for $\lambda=0.1$ and $q_{0}=0.8$ are presented in Fig. 5. The picture in Fig. 5 (b) is similar to that in Fig. 4 (b), which means that the particle redistribution weakly depends on the particle pitch angle when precession is slow. In contrast to this, the increase of the pitch angle in the presence of strong conventional precession enhances the particle redistribution, as follows from Fig. 5 (a). In addition, in Fig. 5 (a) we observe a chain of secondary islands, which partly lies outside the mixing region due to the finite orbit width. Hence, we can conclude that the crash considerably

redistributes toroidally passing particles, at least, when $q_{0} \lesssim 0.9$ or when $q_{0}>0.9$ and precession is weak.

In order to see whether the particle redistribution in accordance to Figs. 3-5 can lead to the escape of the particles to the wall, we have investigated the unperturbed particle motion with the ORBIT code. The results are summarized in Tables I and II. It follows from these tables that particles with the considered pitch angles $(0.1 \leq \lambda \leq 0.58)$ are not confined when $r_{\min } / a \sim 0.4-0.5$, where $r_{\min }$ is the minimum radius of reached by the particle during its orbital motion. This implies that in the considered case of $r_{\text {mix }} / a \sim 0.6-0.7$ the particles can be lost as a result of the crash-induced stochastic motion to the region where $r_{\min } / a \sim 0.4-0.5$

\section{SUMMARY AND CONCLUSIONS}

We have evaluated the change of the beam-plasma fusion reactivity during an MHD event for various patterns of the redistribution of the beam and plasma particles. The result was that if no beam ions are lost, the change of the reactivity is considerably less than the drops of the neutron yield observed during sawtooth crashes in NSTX. This implies that sawtooth oscillations in NSTX are accompanied by a loss of a considerable fraction of the beam ions. 
In particular, a derived equation for the change of the fusion reactivity predicts a $30 \%$ loss of the beam ions in the case when the radial profile of the beam after the crash is flat and the neutron yield drops by a factor of two.

In order to explain the strong influence of sawtooth crashes on energetic ions, we analyzed possible mechanisms of this influence and made a numerical simulation of the motion of beam ions during a sawtooth crash in NSTX.

With this purpose, we evaluated the characteristic times of the beam ion motion. It was concluded that the $\omega_{\phi} / \omega_{\theta}=m / n=1 / 1$ resonance plays an important role unless the toroidal precession is so strong that the island width becomes very small. The resonance adiabatic invariant describing the bounce-averaged particle motion near the 1/1 resonance was derived. Based on this invariant, a general picture of the motion of the toroidally passing particles in the crash field with relatively small amplitude (when the particle motion is regular) was elucidated. A conclusion was drawn that the motion along the resonance islands can lead to significant losses of beam ions during sawteeth when the island width and the mixing radius are sufficiently large.

A numerical study of the motion of $80-\mathrm{keV}$ deuterons during a sawtooth crash in the NSTX shot \#104505 was carried out by the OFSEF code. The latter was modified to model the precession better: the precession of passing particles is very sensitive to the plasma shaping, $\beta$, the radial profile of the plasma pressure etc. The precession in MHD-quiescent states preceding sawtooth crashes was calculated with the ORBIT code. However, this was not sufficient to know the precession during the crashes because of the uncertainty with the contribution of large pressure gradients during the field line reconnection. Therefore, the OFSEF calculations were carried out for two cases: first, with the term modeling the conventional precession and based on the ORBIT results (which overestimates such precession); second, without the mentioned term (which underestimates such precession). It was found that the particle motion can become stochastic in both cases. The most probable mechanism of the stochasticity is the overlap of resonance islands, in particular, secondary islands near the separatrix of the $1 / 1$ resonance and islands produced through the nonlinear mechanism described in Ref. 15 (the particles in the near-axis region are "small-action particles", which can be stochastized in the presence of a monochromatic $m=n=1$ perturbation $^{15}$ ).

The model precession term reduces the stochastic region. This can be explained by the fact that strong toroidal precession diminishes the width of resonance islands, which prevents 
them from overlap. Because the perturbation amplitude decreases with $\left(1-q_{0}\right)$, the role of stochasticity is larger for lower $q_{0}$. In particular, when $q_{0}=0.8$, the calculation without the model term predicts almost completely stochastic motion of toroidally passing particles; with this term, the motion of many particles is stochastic, too, but a considerable number of particles moves regularly along the resonance island. When $q_{0}$ approaches unity, the particle motion is mainly regular, but the resonance island is significant only in the absence of the model term; in the contrary case, the particle orbits are weakly deflected from the $r=$ const flux surfaces.

It follows from our numerical simulations of the particle motion in the NSTX shot \#104505 that the crash results in a flattening of the beam ion distribution and a loss of the particles that enter the loss region. The latter was calculated with the code ORBIT.

\section{Acknowledgments}

One of the authors (Ya.K.) acknowledges the hospitality of the Princeton Plasma Physics Laboratory. The authors thank E. D. Fredrickson and S. S. Medley for providing some data about the NSTX shot \# 104505.

The research described in this work was made possible in part by the Award No. UP22419-KV-02 of the U.S. Civilian Research \& Development Foundation and the Government of Ukraine and the U.S. Department of Energy under contract number DE-AC02-76-CH03073.

\section{APPENDIX A: ORBITAL MOTION OF BEAM IONS IN NSTX: CHARACTER- ISTIC FREQUENCIES, PRECESSION, ESCAPING PARTICLES}

In this Appendix we describe the results of calculations of the orbital motion of $80-\mathrm{keV}$ deuterium ions in the shot \# 104505. The calculations were carried out with the Hamiltonian guiding-center code ORBIT. ${ }^{4,5}$

Figure 6 shows the dependence of the calculated ratio of $\omega_{\phi} / \omega_{\theta}$ on the average radial position of the particle, $\langle r\rangle=\oint d t r / \tau_{b}$, for various magnitudes of the normalized magnetic moment, $\lambda=\mu B_{0} / \mathcal{E}$. Here the radial coordinate, $r$, is defined as the equatorial half-width of the corresponding flux surface. One can see that $\omega_{\phi} / \omega_{\theta}$ significantly differs from $q$ shown in Fig. 2, which shows that the toroidal precession is rather strong. 
It is of interest to compare the precession calculated by ORBIT with analytical predictions. It was shown recently ${ }^{22}$ that in the near-axis region, where $q$ is approximately constant and the pressure profile is approximately parabolic, the precession frequency of well circulating particles (for which $\lambda \epsilon \ll 1-\lambda$ ) is

$$
\omega_{D} \equiv \omega_{\phi}-q_{0} \omega_{\theta}=\xi \frac{v^{2}}{R_{0}^{2} \omega_{B 0}},
$$

where

$$
\begin{aligned}
\xi & =\frac{q_{0}}{k}\left[\frac{1-\frac{3}{2} \lambda+\frac{3}{8} \lambda^{2}}{1-\lambda}-(2-\lambda) \frac{4 k^{2}+1}{3 k^{2}+1}-\frac{\lambda}{2} \tilde{\alpha}_{p}-(2-\lambda) \frac{2 q_{0}^{2} \tilde{\alpha}_{p}}{3 k^{2}+1}\right. \\
& \left.+(2-\lambda) \frac{k^{2}+1}{4 q_{0}^{2}}-(2-\lambda) \frac{k^{2}-1}{3 k^{2}+1} \frac{3 \delta R_{0}}{2 r}\right]
\end{aligned}
$$

is a dimensionless parameter depending on the magnetic configuration and $\lambda, \delta$ is the triangularity of the flux surfaces, $\tilde{\alpha}_{p}=-R_{0}^{2} d \hat{\beta} / d\left(r^{2}\right), \hat{\beta}=8 \pi p / B_{0}^{2}$. The first two terms in the square brackets in Eq. (A2) represent the joint effect of toroidicity (including the Shafranov shift not associated with the plasma pressure) and the elongation of the flux surfaces. The third term shows the effect of the plasma diamagnetism or, in other words, of the radial gradient of $B$ arising from the plasma pressure gradient due to the pressure balance. The fourth term represents the effect of the Shafranov shift caused by the plasma pressure. Finally, the fifth and sixth terms are associated with the plasma paramagnetism and triangularity, respectively.

Table III contains $\xi$ calculated with ORBIT for the moments $t=0.155 \mathrm{~s}$ and $t=0.185 \mathrm{~s}$ and the same quantities following from Eq. (A2), including the contributions from separate terms of this equation. We selected for this table the data concerning semi-trapped particles (i.e., circulating particles not encircling the magnetic axis) with $\lambda=0$. The reason was that the deviation from the magnetic axis is the smallest for such particles; therefore, one can expect that Eqs. (A1) and (A2) are best applicable for them. Note that the calculations for different magnitudes of $\lambda$ in the range of $0 \leq \lambda \leq 0.6$ gave almost the same results as the dependence of $\xi$ on $\lambda$ is weak. We observe that $\xi$ calculated with ORBIT is not constant for semi-trapped particles, which cannot be the case in the adopted analytical approximation. In addition, the analytical theory yields negligible precession for $t=0.185 \mathrm{~s}$ in contrast to $\xi=-0.3 \div-0.45$ given by ORBIT. Detailed analysis shows that these disagreements between the analytical and numerical results are explained mainly by the fact that the pressure profile on the radial interval occupied by the considered orbits strongly deviates 
from the parabolic shape assumed in the analytical theory (the radial derivative of $p$ even changes the sign near the magnetic axis for $t=0.185 \mathrm{~s}$ ).

One can infer from Table III that precession during a magnetic reconnection, when the plasma is not axisymmetric, can be predicted only with large uncertainty. The reason for this is the relatively large contribution to precession from the pressure-produced Shafranov shift. The other terms of Eq. (A2) are determined by the general geometrical properties of the configuration and, therefore, can be assumed to be weakly affected by a magnetic reconnection (note that the poloidal magnetic field is rather weakly perturbed by a sawtooth $\operatorname{crash} ;{ }^{8}$ the relative perturbation of the toroidal magnetic field in a pressureless plasma is even weaker). In contrast to them, the terms associated with the plasma pressure can be expected to change drastically during a plasma mixing. This may result in a difference between the precession in the unperturbed axisymmetric plasma and the precession during a reconnection event.

We also used ORBIT to estimate the size of the loss cone in the considered shot. With this aim, orbits that touch the plasma boundary at the outermost point were calculated for various $\lambda$. The results are presented in Tables I and II. One can see that the minimum radial coordinates of the marginally confined circulating particles are typically below $0.5 a$, which means that the magnetic reconnections that reach $r=0.5 a$ can well expel the particles to the wall. These calculations also revealed that trapped particles (i.e., the particles that change the sign of $v_{\|}$in the course of their motion) are confined only for $\lambda>1$.

\section{APPENDIX B: ADIABATIC INVARIANT OF RESONANT GUIDING-CENTER MOTION}

The derivation procedure in this Appendix is rather similar to that in Ref. 19. The only difference is that here we are not interested in cyclotron resonance and study the particle motion in the guiding-center approximation.

We proceed from the following guiding-center Lagrangian: ${ }^{23}$

$$
\mathcal{L}\left(\mathbf{x}, v_{\|}, \dot{\mathbf{x}}\right)=\frac{e}{c} \mathbf{A}(\mathbf{x}) \cdot \dot{\mathbf{x}}+\frac{M v_{\|}}{B} \mathbf{B}(\mathbf{x}) \cdot \dot{\mathbf{x}}-(\mathcal{E}+e \Phi)
$$

where $\mathbf{x}$ is the particle position, $\mathbf{A}$ and $\Phi$ are the vector and scalar potentials of the electromagnetic field, respectively; $\mathcal{E}=M v_{\|}^{2} / 2+\mu B(\mathbf{x})$ is considered as a function of $\mathbf{x}$ and 
$v_{\|}$. Note that here we have omitted the term responsible for the gyrophase evolution (which does not affect the motion of the guiding center) and treat $\mu$ as a constant parameter. Thus, the number of the degrees of freedom of the system has decreased to two.

We present the magnetic field as a sum of the axisymmetric equilibrium field and the perturbation: $\mathbf{B}=\mathbf{B}_{(0)}+\tilde{\mathbf{B}}$. Neglecting the equilibrium electric field, we choose the gauge so that $\Phi$ in the unperturbed state is zero. The unperturbed motion has a sufficient set of constants of motion ( $\mathcal{E}$ and the toroidal canonical angular momentum). Therefore, the Lagrangian of the unperturbed guiding-center motion,

$$
\mathcal{L}_{(0)}\left(\mathbf{x}, v_{\|}, \dot{\mathbf{x}}\right)=\frac{e}{c} \mathbf{A}_{(0)}(\mathbf{x}) \cdot \dot{\mathbf{x}}+\frac{M v_{\|}}{B_{(0)}} \mathbf{B}_{(0)}(\mathbf{x}) \cdot \dot{\mathbf{x}}-\mathcal{E},
$$

can be written in the action-angle form:

$$
\mathcal{L}_{(0)}=J_{1} \dot{\varsigma}_{1}+J_{2} \dot{\varsigma}_{2}-\mathcal{E}\left(J_{1}, J_{2}\right)
$$

where $\varsigma_{1}$ and $\varsigma_{2}$ are the poloidal and toroidal canonical angles, respectively, which depend linearly on time along the trajectories: $\dot{\varsigma}_{i}=\omega_{i}\left(J_{1}, J_{2}\right), i=1,2 ; \omega_{1}=\omega_{\theta} ; \omega_{2}=\omega_{\phi} ; J_{1}$ and $J_{2}$ are the corresponding canonically conjugate actions. One can show ${ }^{14}$ that $J_{1}=J_{\theta}$ is given by Eq. (28), $J_{2}=J_{\phi}$, and the canonical angles are given by

$$
\varsigma_{1}=\omega_{\theta} \int_{0}^{\theta} d \theta / \dot{\theta}, \quad \varsigma_{2}=\phi-v\left(\varsigma_{1}\right),
$$

where $v \equiv \int_{0}^{\varsigma_{1}} d \varsigma_{1}\left(\dot{\phi}-\omega_{\phi}\right) / \omega_{\theta}$.

The main idea of our further calculation is the following. Studying the perturbed motion in the vicinity of a separate resonance, $n_{1} \omega_{1}+n_{2} \omega_{2}=0$, where $n_{1}$ and $n_{2}$ are certain integers, we can divide the variables into "slow" variables (the actions and the "resonance" combination $n_{1} \varsigma_{1}+n_{2} \varsigma_{2}$ ) and "fast" ones. In our case, there is only one fast variable left, and any linearly independent combination of the angles can be chosen as this variable (we will take the poloidal angle, $\varsigma_{1}$, for this purpose). We will average the motion by the fast variable, which, in effect, is bounce averaging. To perform the averaging, we will employ the method of Lie transformations of the Lagrangian, ${ }^{24}$ looking for such a coordinate transformation that the transformed Lagrangian is independent on the fast variable to required order. Then the quantity that is canonically conjugate to the new fast coordinate is an approximate constant of motion - that is, an adiabatic invariant. 
To implement this program, we introduce new canonical angular coordinates by the linear transformation

$$
\alpha=Q \varsigma,
$$

where $\boldsymbol{\varsigma}=\operatorname{col}\left(\varsigma_{1}, \varsigma_{2}\right), \boldsymbol{\alpha}=\operatorname{col}\left(\alpha_{1}, \alpha_{2}\right)$ is the vector of the new angles, and

$$
\mathrm{Q}=\left\|\begin{array}{cc}
n_{1} & n_{2} \\
1 & 0
\end{array}\right\| .
$$

One can see that the coordinate $\alpha_{1}$ is chosen so that it is constant at the considered resonance. Instead of the Lagrangian, it will be convenient to use the Lagrangian differential form $\gamma_{(0)}=\mathcal{L}_{(0)} d t$, which in the new coordinates takes the form

$$
\gamma_{(0)}=\mathbf{I} \cdot d \boldsymbol{\alpha}-\mathcal{E} d t
$$

where $\mathbf{I}=\left(\mathrm{Q}^{T}\right)^{-1} \mathbf{J}, \mathbf{J}=\operatorname{col}\left(J_{1}, J_{2}\right)$. Below we will consider $I_{1}$ and $\mathcal{E}$ as independent variables and $I_{2}$ as a function of the $I_{1}$ and $\mathcal{E}$ (this choice could be different).

Calculating the difference $\mathcal{L}-\mathcal{L}_{(0)}$ to first order in the perturbation amplitude from Eqs. (B1) and (B2), we see that the first-order correction to $\gamma \equiv \mathcal{L} d t$ is

$$
\tilde{\gamma}=\frac{e}{c} \tilde{\mathbf{A}} \cdot d \mathbf{x}+M \frac{v_{\|}}{B} \tilde{\mathbf{B}} \cdot d \mathbf{x}-M \frac{v_{\|}}{B^{2}} \tilde{B} \mathbf{B} \cdot d \mathbf{x}-\mu \tilde{B} d t-e \Phi d t
$$

where $\tilde{B}=\mathbf{B} \cdot \tilde{\mathbf{B}} /(2 B)$ is the perturbation of $B$.

Now we will use the assumption that $\alpha_{1}$ is a slow variable near the resonance. Varying Eq. (B7) to obtain the Euler-Lagrange equations, it is easy to find the frequencies of the unperturbed motion. In particular, we obtain

$$
\dot{\alpha}_{1}=-\left(\frac{\partial I_{2}}{\partial \mathcal{E}}\right)^{-1} \frac{\partial I_{2}}{\partial I_{1}} .
$$

We conclude that $\partial I_{2} / \partial I_{1}$ is small (and vanishes at the exact resonance). Therefore, we split $I_{2}$ into two parts, $I_{2}=I_{20}(\mathcal{E})+\varepsilon I_{21}\left(I_{1}, \mathcal{E}\right)$, where $I_{20}(\mathcal{E})$ is $I_{2}$ with the argument $I_{1}$ taken at the exact resonance, $\varepsilon$ is the ordering parameter (in the end we will set $\varepsilon=1$ to obtain physically meaningful results). The perturbation is also assumed to be $\sim \varepsilon$. We obtain the following presentation for $\gamma$ :

$$
\gamma=\gamma_{0}+\varepsilon \gamma_{1}
$$

where

$$
\gamma_{0}=I_{1} d \alpha_{1}+I_{20}\left(I_{3}, \mathcal{E}\right) d \alpha_{2}-\mathcal{E} d t
$$




$$
\gamma_{1}=I_{21}\left(I_{1}, I_{3}, \mathcal{E}\right) d \alpha_{2}+\tilde{\gamma}
$$

Note that all slow variables $\left(I_{1}, \mathcal{E}\right.$, and $\left.\alpha_{1}\right)$ are constants of motion of the Euler-Lagrange equations resulting from $\gamma_{0}$.

Now we proceed to the averaging procedure. Following Ref. 24, we look for a Lie transformation $\overline{\mathbf{z}}=\exp (\varepsilon \mathbf{G}) \mathbf{z}$ that makes the differential form independent on fast variables, where $\mathbf{z}=\left(\alpha_{1}, \alpha_{2}, I_{1}, \mathcal{E}, t\right)$ is the vector of the variables, $\overline{\mathbf{z}}$ is the vector of the corresponding new variables free of rapid oscillations to the first order, $\mathbf{G}$ is the transformation generator. The components of the differential form $\gamma_{1}$ in the new variables look as follows: ${ }^{24}$

$$
\bar{\gamma}_{1 j}=\gamma_{1 j}-G^{i} w_{i j}+\partial_{j} S
$$

where $S$ is an arbitrary function of $\mathbf{z}$; subscripts and superscripts denote co- and contravariant components, respectively; $w_{i j}=\partial_{i} \gamma_{0 j}-\partial_{j} \gamma_{0 i}$ are the Lagrange brackets among the coordinates $\mathbf{z}$ in the system $\gamma_{0}$ (note that perturbations of the magnetic field modify the simplectic structure of the phase space and, in particular, the Lagrange brackets); and summation over repeating indices is implied. Then the averaging reduces to solving Eq. (B13) for $G^{i}$ and $S$, so that $\bar{\gamma}_{1 j}$ have the desired form. Specifically, we demand that all $\bar{\gamma}_{1 j}$ should vanish except for $j=\alpha_{2}$ and that $\bar{\gamma}_{1 \alpha_{2}}$ should not depend on the fast variable $\alpha_{2}$.In addition, we set $G^{t}=0$ (the time variable should not be modified by the transformation). The procedure of solving is omitted here because it is very similar to that in the case of three degrees of freedom, which was presented in Ref. 19 (Appendix B). We obtain:

$$
\bar{\gamma}_{1 \alpha_{2}}=\frac{1}{2 \pi n_{2}} \oint_{L\left(\alpha_{1}, J_{\phi}, \mathcal{E}\right)} \gamma_{1},
$$

where $L\left(\alpha_{1}, J_{\phi}, \mathcal{E}\right)$ is the closed loop determined by the equations $-n_{2} \pi \leq \varsigma_{1} \leq n_{2} \pi$, $\varsigma_{2}=\left(\alpha_{1}-n_{1} \varsigma_{1}\right) / n_{2}, J_{\phi}=$ const, $\mathcal{E}=$ const. This loop is, in fact, an unperturbed particle trajectory with the toroidal frequency, $\omega_{2}$, modified to satisfy the resonance condition exactly.

Using Eqs. (B11), (B12), and (B14), we obtain the averaged Lagrangian:

$$
\mathcal{L}=\bar{I}_{1} \cdot \dot{\bar{\alpha}}_{1}+I\left(\bar{\alpha}_{1}, \bar{I}_{1}, \overline{\mathcal{E}}\right) \dot{\bar{\alpha}}_{2}-\overline{\mathcal{E}}
$$

where

$$
I\left(\bar{\alpha}_{1}, \bar{I}_{1}, \overline{\mathcal{E}}\right)=\bar{I}_{2}+\frac{1}{2 \pi n_{2}} \oint_{L\left(\alpha_{1}, J_{\phi}, \mu, \mathcal{E}\right)} \tilde{\gamma}
$$


is a constant of motion of the averaged system (we have already set $\varepsilon=1$ ). On the other hand, the difference between the coordinates $\left(\bar{\alpha}_{1}, \bar{\alpha}_{2}, \bar{I}_{1}, \overline{\mathcal{E}}\right)$ and $\left(\alpha_{1}, \alpha_{2}, I_{1}, \mathcal{E}\right)$ can result only in adiabatic variations of the "non-averaged" variables $\left(\alpha_{1}, \alpha_{2}, I_{1}, \mathcal{E}\right)$. Due to this, $I$ given by Eq. (B16) and considered as a function of $\left(\alpha_{1}, I_{1}, \mathcal{E}\right)$ is a new adiabatic invariant - the resonance adiabatic invariant that we have intended to obtain. Substituting $\gamma=\mathcal{L} d t$ from Eq. (B1) and expressing $I_{2}$ in terms of $\mathbf{J}$, we obtain the following expression for the resonance adiabatic invariant:

$$
\begin{aligned}
I & =J_{\theta}\left(J_{\phi}, \mathcal{E}\right)-\frac{n_{1}}{n_{2}} J_{\phi} \\
& +\frac{1}{2 \pi n_{2}} \oint_{L\left(\alpha_{1}, J_{\phi}, \mathcal{E}\right)}\left[d \mathbf{x} \cdot\left(\frac{e}{c} \tilde{\mathbf{A}}+M \frac{v_{\|}}{B} \tilde{\mathbf{B}}-M \frac{v_{\|}}{B^{2}} \mathbf{B} \tilde{B}\right)-d t(\mu \tilde{B}+e \Phi)\right] .
\end{aligned}
$$

Equation (B17) is a generalization of a previous result, ${ }^{14}$ which was obtained for perturbations that do not affect $B$, to the case of general time-independent perturbations. In the particular case of the resonance $\omega_{\phi} / \omega_{\theta}=1 / 1$ the obtained resonance is reduced to Eq. (27).

1 M. Ono, S. M. Kaye, Y.-K. M. Peng et al., Nucl. Fusion 40, 557 (2000).

2 S. S. Medley and A. L. Roquemore "Neutral Particle Analyzer Measurements of Ion Behavior in NSTX", NSTX Results Review, PPPL, September 19-20, 2001.

3 Ya. I. Kolesnichenko, V. V. Lutsenko, R. B. White, and Yu. V. Yakovenko, Nucl. Fusion 40, 1325 (2000).

4 R. B. White and M. S. Chance, Phys. Fluids 27, 2455 (1984).

5 R. B. White, Phys. Fluids B 2, 845 (1990).

6 Ya. I. Kolesnichenko and Yu. V. Yakovenko, Nucl. Fusion 32, 449 (1992).

7 Ya. I. Kolesnichenko, Nucl. Fusion 20, 727 (1980).

8 B. B. Kadomtsev, Sov. J. Plasma Phys. 1, 389 (1976).

9 Ya. I. Kolesnichenko, Yu. V. Yakovenko, D. Anderson, M. Lisak, and F. Wising, Phys. Rev. Lett. 68, 3881 (1992).

10 D. Anderson, Ya. I. Kolesnichenko, M. Lisak, F. Wising, and Yu. V. Yakovenko, Nucl. Fusion 34, 217 (1994).

11 Ya. I. Kolesnichenko, V. V. Lutsenko, R. B. White, and Yu. V. Yakovenko, Phys. Lett. A 287, 131 (2001). 
12 Ya. I. Kolesnichenko and Yu. V. Yakovenko, Nucl. Fusion 36, 159 (1996).

13 Ya. I. Kolesnichenko, V. V. Lutsenko, Yu. V. Yakovenko, and G. Kamelander, Phys. Plasmas 4, 2544 (1997).

14 Ya. I. Kolesnichenko, V. V. Lutsenko, R. B. White, and Yu. V. Yakovenko, Phys. Plasmas 5, 2963 (1998).

15 Ya. I. Kolesnichenko, V. V. Lutsenko, R. B. White, and Yu. V. Yakovenko, Phys. Rev. Lett. 84, $2152(2000)$.

16 Ya. I. Kolesnichenko, V. S. Marchenko, and R. B. White, Phys. Plasmas 8, 3143 (2001).

17 Ya. I. Kolesnichenko, V. V. Lutsenko, V. S. Marchenko, R. B. White, and Yu. V. Yakovenko, in Fusion Energy 2002, 19th Conference Proceedings, Lyon, 2002 (International Atomic Energy Agency, Vienna, 2003), Report TH/P3-15.

18 Yu. V. Yakovenko, in 1998 International Congress on Plasma Physics combined with 25th European Physical Society Conference on Controlled Fusion and Plasma Physics, Praha, 1998, Europhysics Conference Abstracts, Vol. 22C (The European Physical Society, Petit-Lancy, 1998), p. 2133-2136.

19 Ya. I. Kolesnichenko, R. B. White, and Yu. V. Yakovenko, Phys. Plasmas 9, 2639 (2002).

20 R. V. Budny, M. G. Bell, A. C. Janos et al., Nucl. Fusion 35, 1497 (1995).

21 E. D. Fredrickson, private communication (2002).

22 Ya. I. Kolesnichenko, R. B. White, and Yu. V. Yakovenko, Phys. Plasmas 10, 1449 (2003).

23 R. G. Littlejohn, J. Plasma Phys. 29, 111 (1983).

24 R. G. Littlejohn, J. Math. Phys. 23, 742 (1982). 


\section{TABLES}

TABLE I: Parameters of marginally confined co-circulating orbits (i.e., the co-circulating orbits for which $R+\rho_{\perp} \approx R_{\max }$ in the outermost point of the orbit, where $\rho_{\perp}=v_{\perp} / \omega_{B}, R_{\max }=148.9 \mathrm{~cm}$ is $R$ at the outermost point of the plasma boundary) for $t=0.155 \mathrm{~s}$.

\begin{tabular}{l|c|c|c|c}
\hline$\lambda$ & $\begin{array}{c}\text { minimum } r / a \\
(r / a \text { for } \theta=\pi)\end{array}$ & \multicolumn{2}{|l|}{ parameters at the outermost point of the orbit $(\theta=0)$} \\
\hline 0.1 & 0.51 & 0.85 & 143.3 & $\rho_{\perp}, \mathrm{cm}$ \\
0.2 & 0.47 & 0.80 & 140.8 & 6.0 \\
0.3 & 0.44 & 0.76 & 138.9 & 8.5 \\
0.4 & 0.40 & 0.72 & 137.2 & 10.3 \\
0.5 & 0.37 & 0.69 & 135.8 & 11.8 \\
0.58 & 0.34 & 0.67 & 134.8 & 13.1 \\
\hline
\end{tabular}

TABLE II: The same as Table I but for $t=0.185 \mathrm{~s}\left(R_{\max }=153.7 \mathrm{~cm}\right)$.

\begin{tabular}{l|c|c|c|c}
\hline$\lambda$ & $\begin{array}{c}\text { minimum } r / a \\
(r / a \text { for } \theta=\pi)\end{array}$ & \multicolumn{2}{|l}{ parameters at the outermost point of the orbit $(\theta=0)$} \\
\cline { 3 - 5 } 0.1 & 0.54 & 0.84 & 147.7 & $\rho_{\perp}, \mathrm{cm}$ \\
0.2 & 0.50 & 0.79 & 145.2 & 6.2 \\
0.3 & 0.47 & 0.75 & 143.3 & 8.7 \\
0.4 & 0.44 & 0.72 & 141.5 & 10.6 \\
0.5 & 0.41 & 0.70 & 140.5 & 12.2 \\
0.58 & 0.38 & 0.67 & 139.3 & 13.6 \\
\hline
\end{tabular}

TABLE III: Comparison of the coefficients $\xi$ calculated by ORBIT and analytically for semi-trapped particles with $\lambda=0$.

\begin{tabular}{l|c|c|l|l|l|l|c}
\hline$t, \mathrm{~s}$ & $\xi$ from ORBIT & $\xi$ from Eq. (A2) & \multicolumn{4}{|l|}{ Contributions from separate terms of Eq. (A2) } \\
\cline { 4 - 8 } & & 1 st and 2nd 3 rd & 4 th & 5 th & 6 th \\
\hline 0.155 & $-1.05 \div-1.45$ & -1.1 & -1.2 & 0 & -0.6 & 0.9 & -0.2 \\
0.185 & $-0.3 \div-0.45$ & 0.0 & -1.1 & 0 & 0.1 & 1.1 & -0.1 \\
\hline
\end{tabular}




\section{FIGURES}
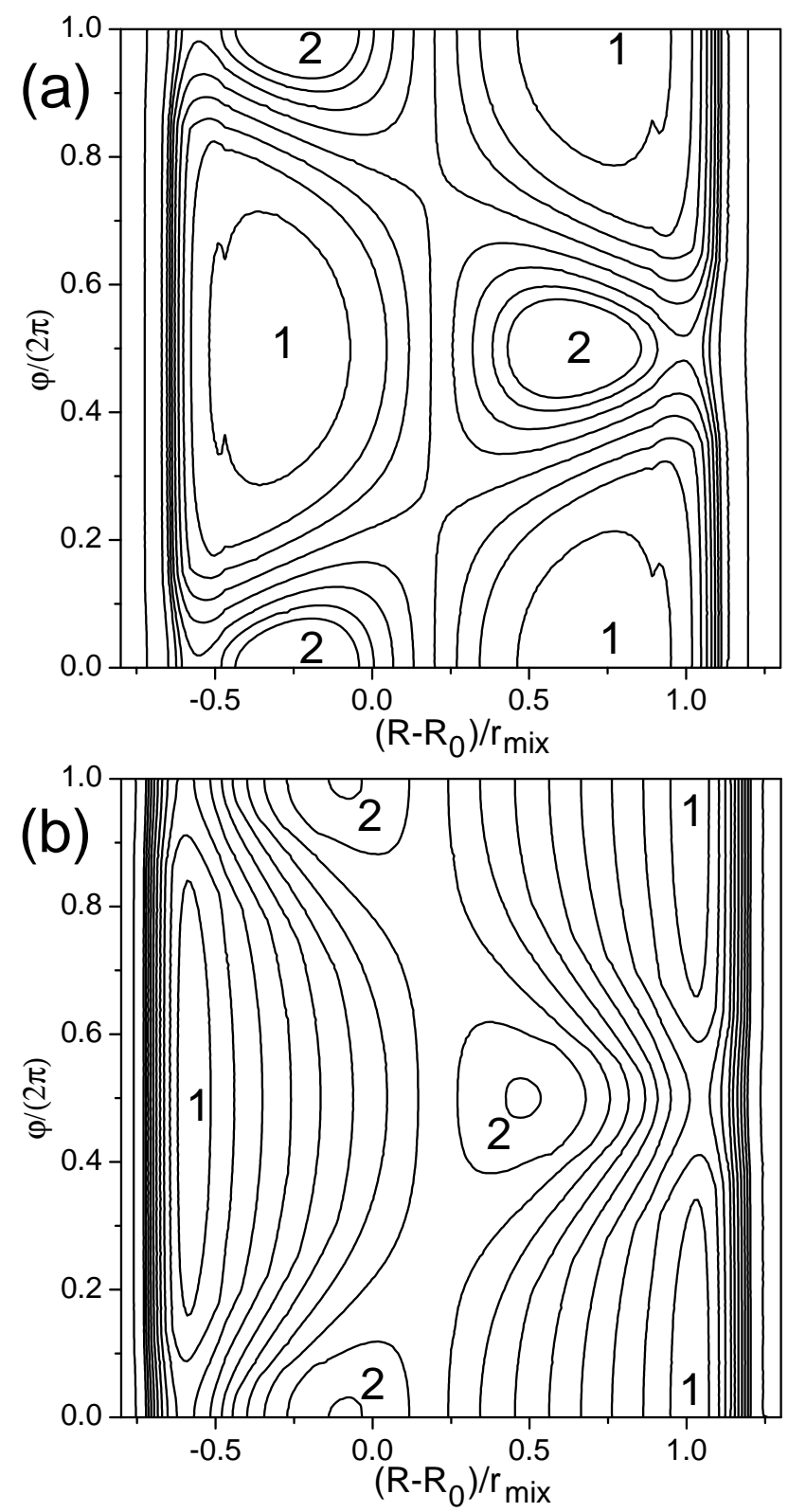

FIG. 1: Crossings of drift surfaces of $80-\mathrm{keV}$ deuterons during a sawtooth crash with the equatorial plane for $\lambda=0.51, \beta_{0}=0.11, R_{0}=100 \mathrm{~cm}, a=68 \mathrm{~cm}, r_{\text {mix }}=50 \mathrm{~cm}, B_{0}=0.36 \mathrm{~T}, q_{0}=0.9, q_{a}=$ 10. (a), $\xi=-0.01$; (b) $\xi=-0.2$. 1, the $1 / 1$ resonance island; 2, displaced central particles. Each drift surface is shown twice, the left and right crossings with the equatorial plane corresponding to the parts of the particle orbits with the minimum and maximum distances from the magnetic axis, respectively. The pictures are obtained with the use of the invariant given by Eq. (27). 


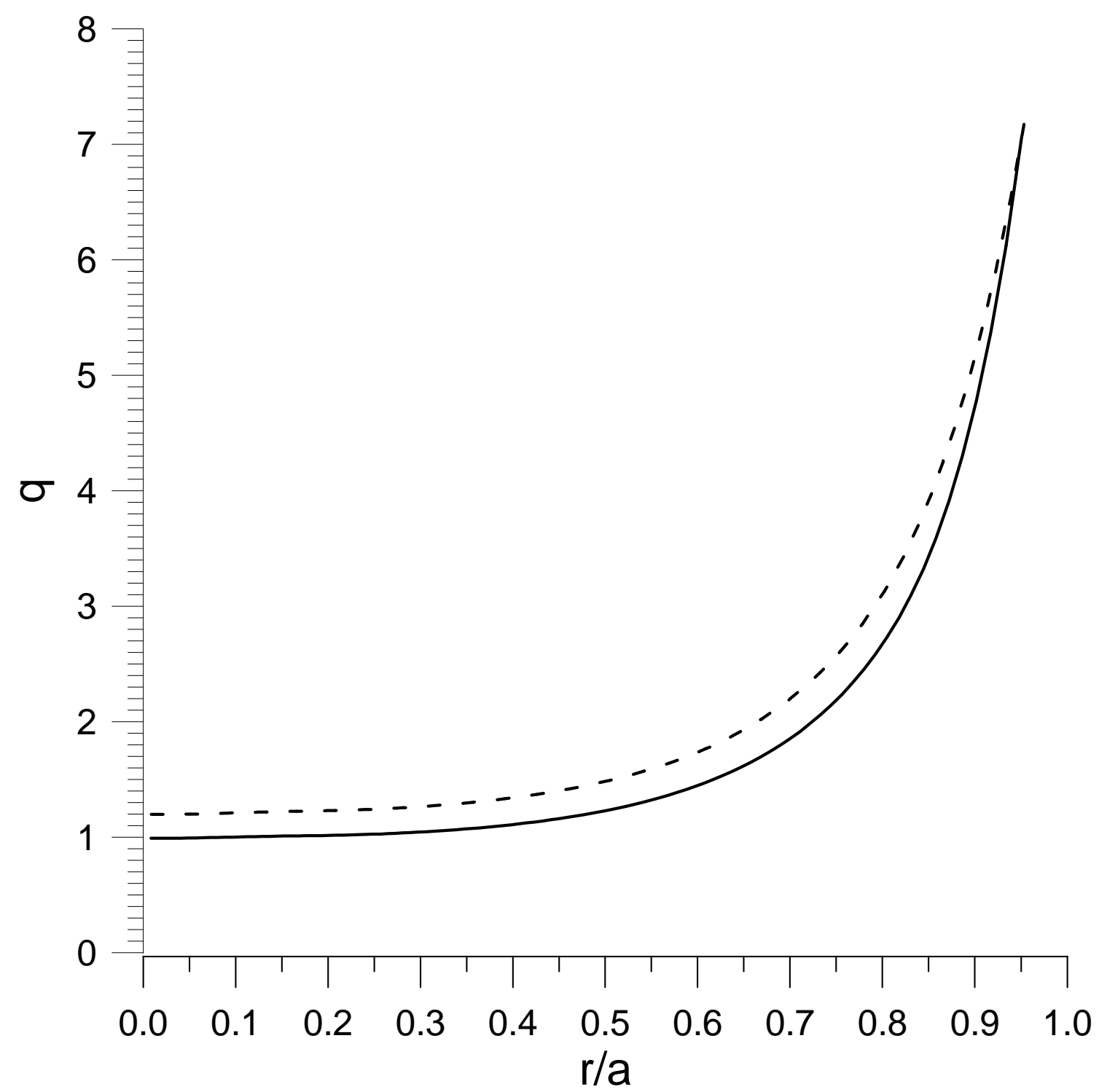

FIG. 2: The $q(r)$ profile calculated by TRANSP for the NSTX shot \# 104505. ${ }^{21}$ Dashed line, $t=0.155 \mathrm{~s} ;$ solid line, $t=0.185 \mathrm{~s}$. 

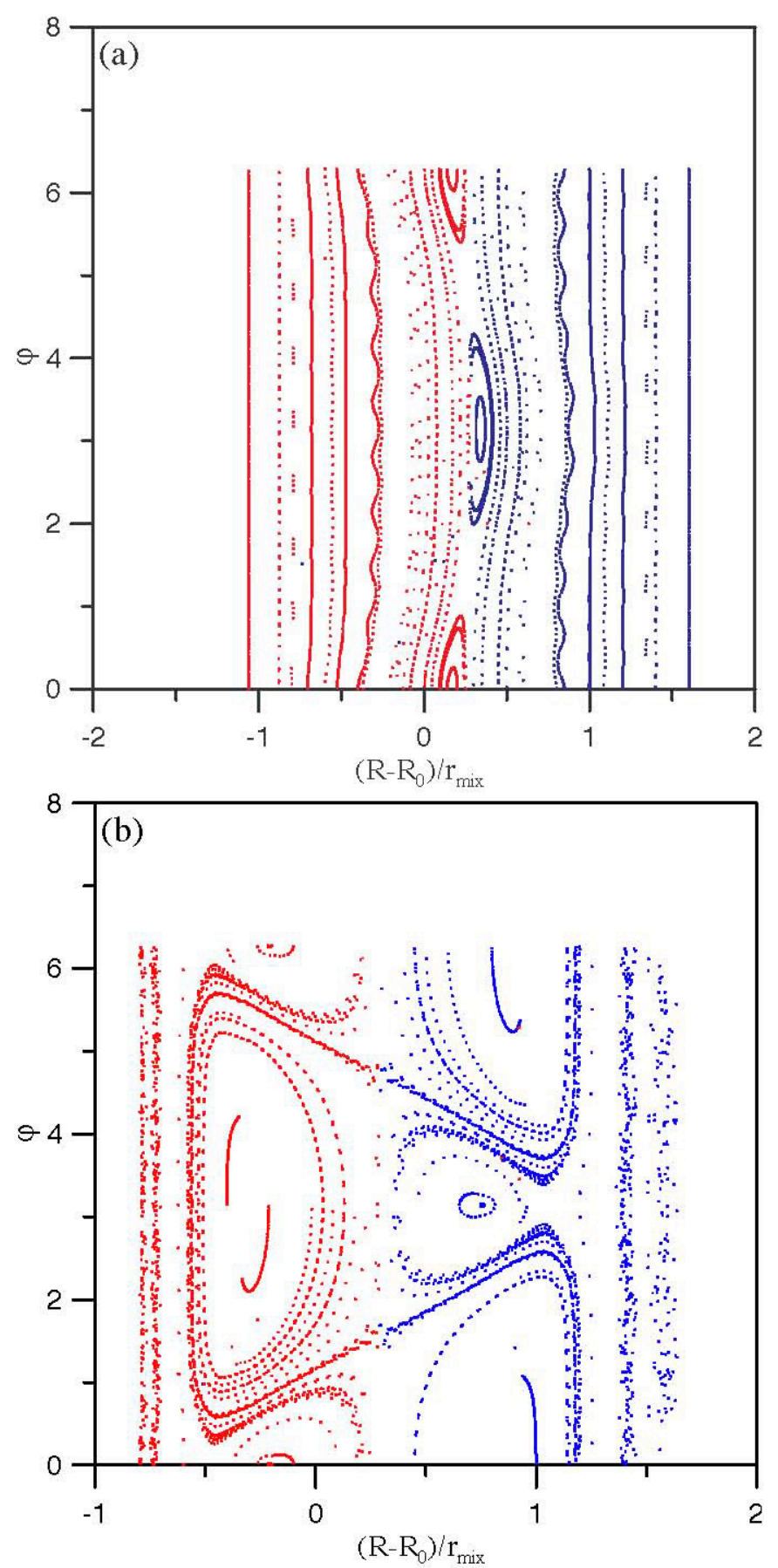

FIG. 3: Poincaré map of the motion of $80-\mathrm{keV}$ ions launched with $v_{\|}>0$ and $\lambda=0.58$ in NSTX for $q_{0}=0.95$ at the nonlinear stage of the sawtooth crash that occurred at $t_{2} \approx 0.19$ : (a) with the conventional precession term; (b) without this term. The blue and red color mark the footprints left at the equatorial plane of the torus when the particles are at the maximum and minimum distances from the magnetic axis, respectively. The pictures are obtained with the use of the code OFSEF. 

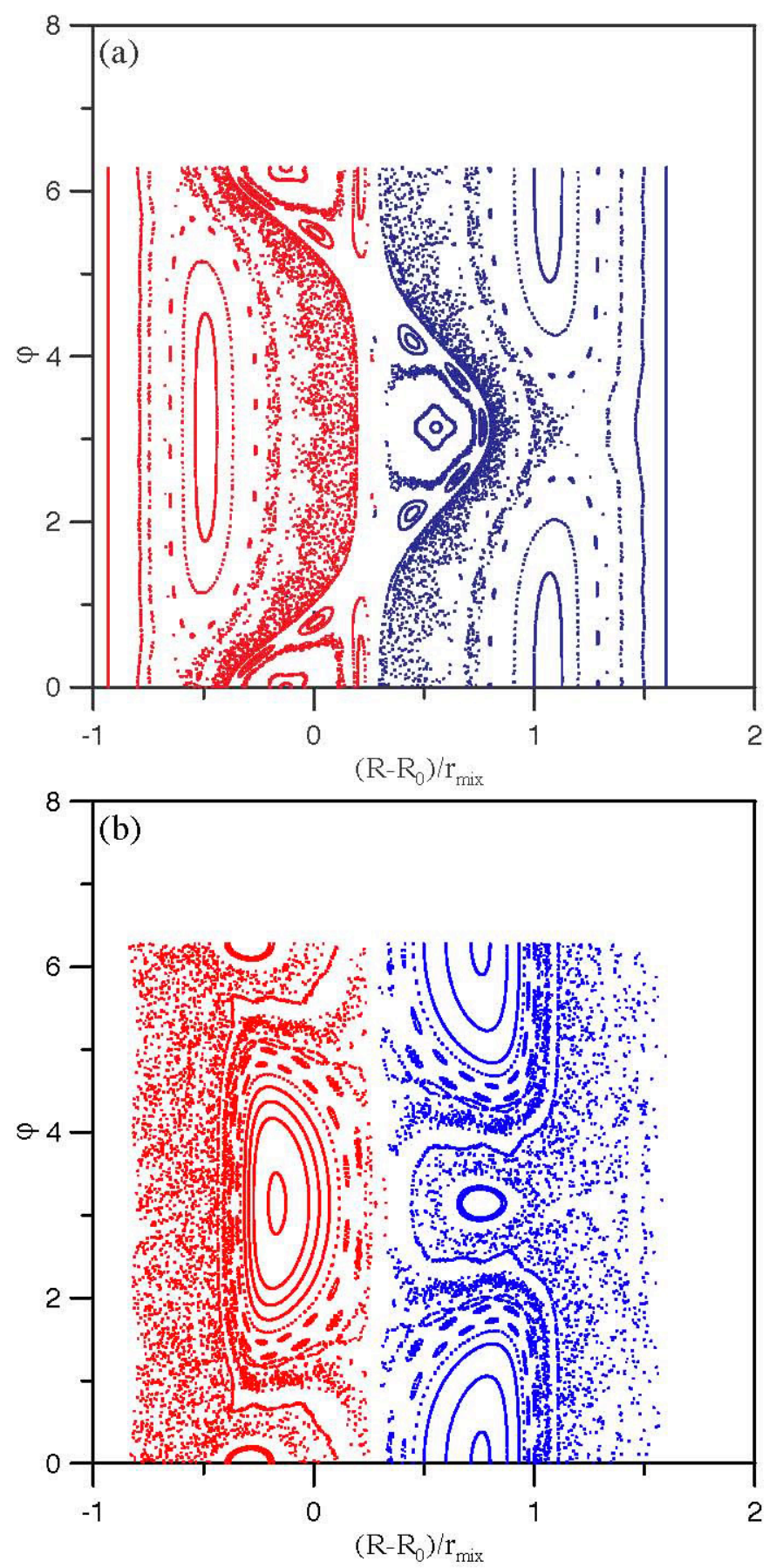

FIG. 4: The same as Fig. 3 but for $q_{0}=0.8$. 

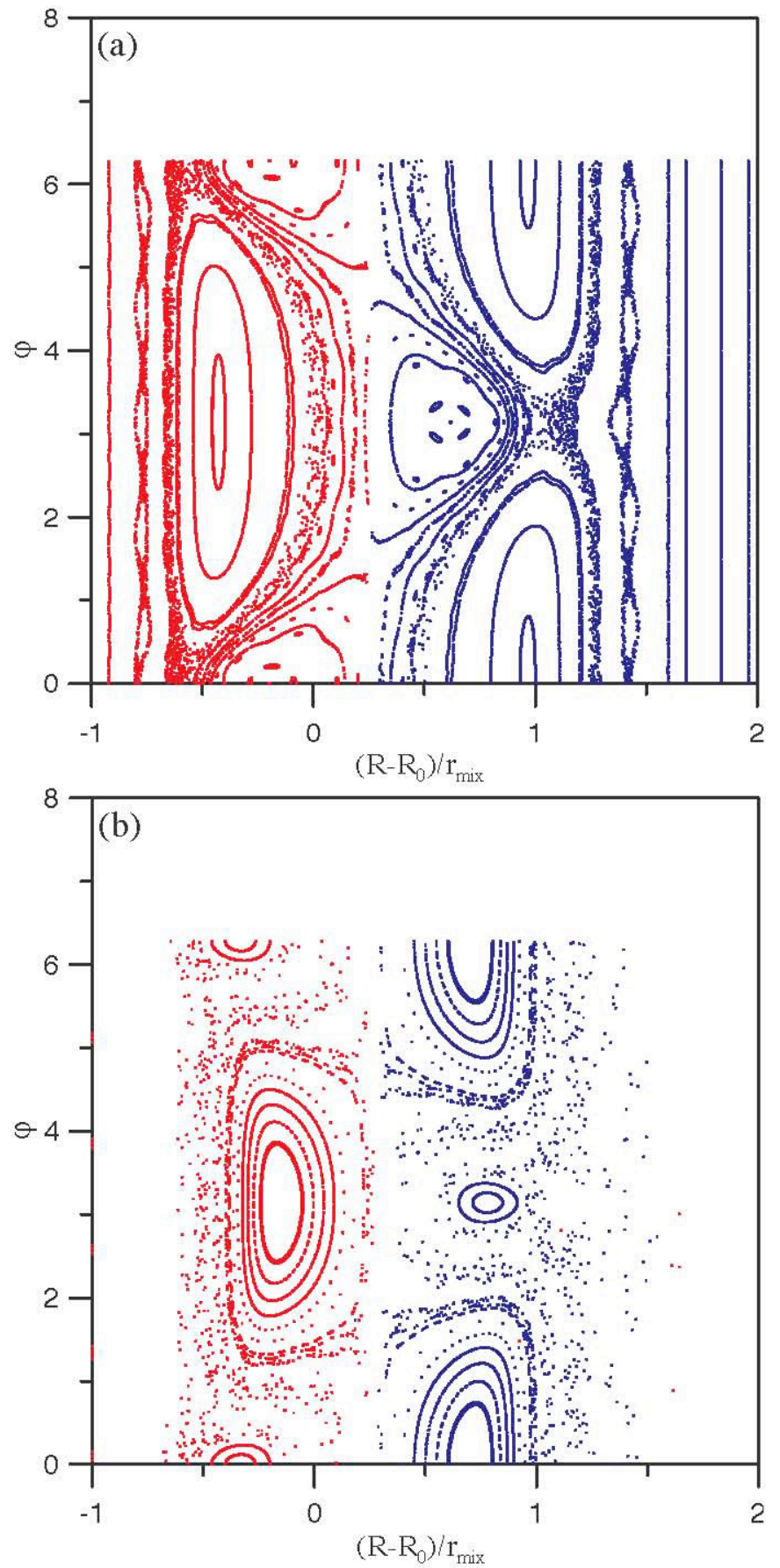

FIG. 5: The same as Fig. 4 but for $\lambda=0.1$. 


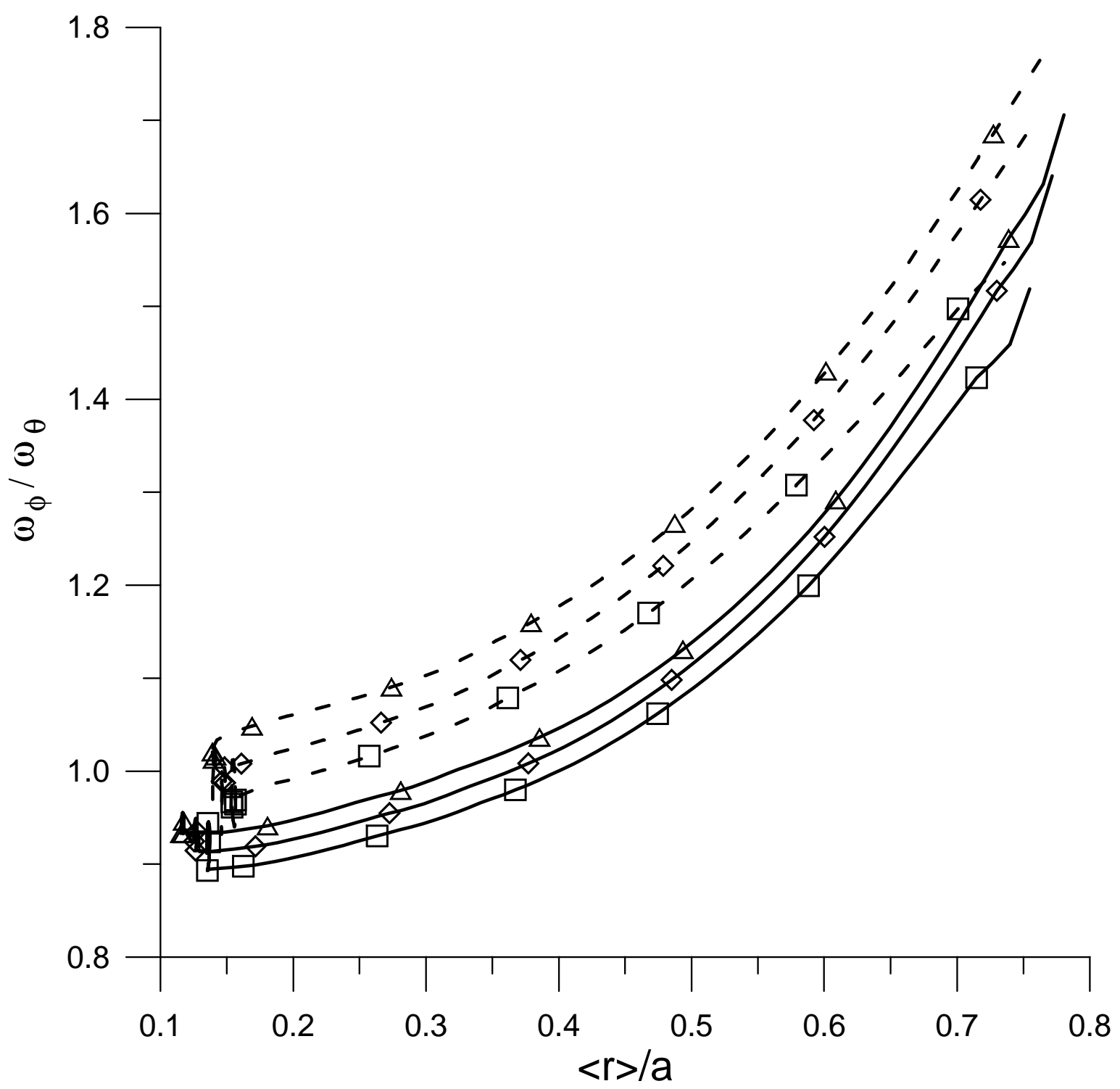

FIG. 6: The radial dependence of $\omega_{\phi} / \omega_{\theta}$ calculated by the code ORBIT for various $\lambda$ in the NSTX shot \# 104505. $\triangle, \lambda=0 ; \diamond, \lambda=0.4 ; \square, \lambda=0.58$. Dashed lines, $t=0.155 \mathrm{~s}$; solid lines, $t=0.185 \mathrm{~s}$. 


\section{External Distribution}

Plasma Research Laboratory, Australian National University, Australia

Professor I.R. Jones, Flinders University, Australia

Professor João Canalle, Instituto de Fisica DEQ/IF - UERJ, Brazil

Mr. Gerson O. Ludwig, Instituto Nacional de Pesquisas, Brazil

Dr. P.H. Sakanaka, Instituto Fisica, Brazil

The Librarian, Culham Laboratory, England

Mrs. S.A. Hutchinson, JET Library, England

Professor M.N. Bussac, Ecole Polytechnique, France

Librarian, Max-Planck-Institut für Plasmaphysik, Germany

Jolan Moldvai, Reports Library, Hungarian Academy of Sciences, Central Research Institute for Physics, Hungary

Dr. P. Kaw, Institute for Plasma Research, India

Ms. P.J. Pathak, Librarian, Institute for Plasma Research, India

Ms. Clelia De Palo, Associazione EURATOM-ENEA, Italy

Dr. G. Grosso, Instituto di Fisica del Plasma, Italy

Librarian, Naka Fusion Research Establishment, JAERI, Japan

Library, Laboratory for Complex Energy Processes, Institute for Advanced Study, Kyoto University, Japan

Research Information Center, National Institute for Fusion Science, Japan

Dr. O. Mitarai, Kyushu Tokai University, Japan

Dr. Jiangang Li, Institute of Plasma Physics, Chinese Academy of Sciences, People's Republic of China

Professor Yuping Huo, School of Physical Science and Technology, People's Republic of China

Library, Academia Sinica, Institute of Plasma Physics, People's Republic of China

Librarian, Institute of Physics, Chinese Academy of Sciences, People's Republic of China

Dr. S. Mirnov, TRINITI, Troitsk, Russian Federation, Russia

Dr. V.S. Strelkov, Kurchatov Institute, Russian Federation, Russia

Professor Peter Lukac, Katedra Fyziky Plazmy MFF UK, Mlynska dolina F-2, Komenskeho Univerzita, SK-842 15 Bratislava, Slovakia

Dr. G.S. Lee, Korea Basic Science Institute, South Korea

Institute for Plasma Research, University of Maryland, USA

Librarian, Fusion Energy Division, Oak Ridge National Laboratory, USA

Librarian, Institute of Fusion Studies, University of Texas, USA

Librarian, Magnetic Fusion Program, Lawrence Livermore National Laboratory, USA

Library, General Atomics, USA

Plasma Physics Group, Fusion Energy Research Program, University of California at San Diego, USA

Plasma Physics Library, Columbia University, USA

Alkesh Punjabi, Center for Fusion Research and Training, Hampton University, USA

Dr. W.M. Stacey, Fusion Research Center, Georgia Institute of Technology, USA

Dr. John Willis, U.S. Department of Energy, Office of Fusion Energy Sciences, USA

Mr. Paul H. Wright, Indianapolis, Indiana, USA 
The Princeton Plasma Physics Laboratory is operated by Princeton University under contract with the U.S. Department of Energy.

\author{
Information Services \\ Princeton Plasma Physics Laboratory \\ P.O. Box 451 \\ Princeton, NJ 08543
}

Phone: 609-243-2750

Fax: 609-243-2751

e-mail: pppl_info@pppl.gov

Internet Address: http://www.pppl.gov 